\title{
An association between fingerprint patterns with blood group and lifestyle based diseases: a review
}

\author{
Vijaykumar Patil $^{1}$ (D) D. R. Ingle ${ }^{1}$
}

Published online: 18 August 2020

(c) Springer Nature B.V. 2020

\begin{abstract}
In the current era of the digital world, the hash of any digital means considered as a footprint or fingerprint of any digital term but from the ancient era, human fingerprint considered as the most trustworthy criteria for identification and it also cannot be changed with time even up to the death of an individual. In the court of law, fingerprint-proof is undeniably the most dependable and acceptable evidence to date. Fingerprint designs are exclusive in each human and the chance of two individuals having identical fingerprints is an exceptional case about one in sixty-four thousand million also the fingerprint minutiae patterns of the undistinguishable twins are different, and the ridge pattern of each fingertip remain unchanged from birth to till death. Fingerprints can be divided into basic four categories i.e. Loop, whorl, arch, and composites, nevertheless, there are more than 100 interleaved ridge and valleys physiognomies, called Galton's details, in a single rolled fingerprint. Due to the immense potential of fingerprints as an effective method of identification, the present research paper tries to investigate the problem of blood group identification and analysis of diseases those arises with aging like hypertension, type 2-diabetes and arthritis from a fingerprint by analyzing their patterns correlation with blood group and age of an individual. The work has been driven by studies of anthropometry, biometric trademark, and pattern recognition proposing that it is possible to predict blood group using fingerprint map reading. Dermatoglyphics as a diagnostic aid used from ancient eras and now it is well established in number of diseases which have strong hereditary basis and is employed as a method for screening for abnormal anomalies. Apart from its use in predicting the diagnosis of disease; dermatoglyphics is also used in forensic medicine in individual identification, physical anthropology, human genetics and medicine. However, the Machine and Deep Learning techniques, if used for fingerprint minutiae patterns to be trained by Neural Network for blood group prediction and classification of common clinical diseases arises with aging based on lifestyle would be an unusual research work.
\end{abstract}

Keywords ABO typing $\cdot$ Blood groups $\cdot$ Fingerprint map reading $\cdot$ Machine learning Deep leaning $\cdot$ Artificial intelligence

Vijaykumar Patil

vijay.patil.karad@gmail.com

D. R. Ingle

dringleus@gmail.com

1 Bharati Vidyapeeth College of Engineering, University of Mumbai, Navi Mumbai, India 


\section{Introduction}

The study of fingerprint patterns was introduced by Dr. Harold Cummins in 1926 but it is already in use before several hundred years ago. Fingerprint patterns have been normally used for identification of an individual. Now a days every organization or even may Government institutes in India, use fingerprint verification to identify everyone uniquely and it also have been used as a biometric modality for gender and age identification. An individual is their own key; behind this catchy principle biometrics have become an attractive alternative to traditional identification methods such as tokens or passwords (Fernandes et al. 2013). Current fingerprint matching methods were started in the sixteenth century. It was Henry Fauld in 1880 who first experimentally proposed the singularity and uniqueness of fingerprint. Herschel (Ravindran et al. 2017) added to the establishment of current fingerprinting identification. In the nineteenth century Sir Francis Galton (McBean et al. 2014) directed broad investigations and ordered the sorts of fingerprints relying on essential example as loops, whorls and arches. It was Cummins (Ferraz et al. 2010) who authored the expression "Dermatoglyphics (derma $1 / 4$ skin, glyphic $1 / 4$ bends), to dermal edge arrangements on the digits, of palms and sole and furthermore demonstrated that edge design are resolved incompletely by heredity or natural impact which produce pressure and strain in their development during fetal life. The fingerprint design whorl might be winding, oval, roundabout or any assortment of a loop and record for around 30\%. Arches are the basic type up till now uncommon (about 5\%). The fingerprint design has edges running from one side to the opposite side of the print without having any re-bend. The term composite is utilized for mix of type example that doesn't fit into any of the above characterization (Azhagiri et al. 2018). Till date, analysts or researches have generally used fingerprint details as perspectives to build up any individuals uniqueness. The ridge patterns have been comprehensively classified into five different kinds called as arch, tented arch, whorl, ulnar and radial loop. An individual can have any of the above type in any of the its fingers. All things considered, dominant part of fingerprints found in populace review shows that 70 percent of the prints are loops, 20-25 percent being whorls though just 5 to 10 percent consider arch or tented arch patterns or designs (Singh and Majumdar 2015). Some examinations done on twins have presumed that monozygotic or indistinguishable twins have comparable however not indistinguishable examples found.

Dermatoglyphics as a diagnostic aid used from ancient eras and now it is well established in number of diseases which have strong hereditary basis and is employed as a method for screening for abnormal anomalies. Fingerprint minutiae patterns of ridges are determined as unique through the combination of genetic and environment factors. Person identification using fingerprint algorithms are well sophisticated and are being established all over the world for security and authentication. The fingerprint also used to classify gender and age group but very few manual attempts have been made to explore relationship between fingerprint patterns with blood group and common clinical diseases like hypertension, type-2 diabetes and arthritis. It will be helpful for anthropologists to predict blood group and classify common clinical diseases than conventional pathological techniques from the fingerprints those are obtained from mined articles using Deep Learning Techniques for early reminder to prevent such common clinical diseases those arises with aging and also crime investigators to minimize the range of the suspects it would be predicted using Deep Neural Network. The dermatoglyphics and its important role in the diagnosis of different diseases like hypertension, type- 2 diabetes and arthritis with genetic bases. Apart from its use in predicting the diagnosis of disease; dermatoglyphics is also used in 
forensic medicine in individual identification, physical anthropology, human genetics and medicine.

\section{Objectives of research}

The research work is aimed in developing the Deep Neural Network algorithms for accurate classification of the fingerprints obtained which include:

- To Enhancement Fingerprint Image During sampling or in data set preparation step fingers of an individual recorded using fingerprint scanner. To enhance the fingerprint images precisely, the research focuses to develop various pre-processing algorithms like-Segmentation, Normalization, Orientation estimation, Ridge frequency estimation, Gabor filter and Binarisation and Thinning etc.

- To Extraction of Features from Fingerprints and Finding Similarity Vector To build similarity vector using features of captured sample images of fingerprint required a feature extraction algorithm. The implementation of the biometric features extraction algorithms needs to extract features like-the ridge count, ridge thickness to valley thickness ratio (RTVTR), white lines count, ridge count asymmetry, minutiae map(MM) orientation collinearity maps(OCM), Gabor Feature maps(GFM), orientation map (OM) for pattern type, 2D wavelet transform (DWT)

- To Predict Blood Group by using Extracted Feature of Fingerprints The unsupervised machine learning technique will apply for classification of blood group which helps to identify relationship patterns of different features of fingerprints with $\mathrm{ABO}$ blood type and then prediction will perform with the application of Machine Learning and Convolutional Neural Network (CNN) technology with the help of rigid frequency count and distance formula to conclude blood group from feature vector.

- To Classify and Analyse lifestyle diseases like hypertension, arthritis and diabetes Normally common clinical diseases arise with the age but, now in current era these are no more only relevant to the age; due to busy schedule or lifestyle of an individual they arise at any stage of life. With the fingerprint images and blood group of an individual, the dataset include the external attributes like age, weight, height, skin color, eyes color, work nature, eating habits (vegetarian or non-vegetarian), region (rural or urban), addiction (if any like drink, smoke), etc.

The rest of the paper is organized as follows. The conceptual background discussed in Sect. 2. The literature review specificity discusses all the methods used in Sect. 3 and the evaluation and discussion included in Sect. 4 which illustrates the summary of different dataset/samples and methodologies used. Finally, in Sect. 5, we conclude the paper.

\section{Conceptual background}

The common types of fingerprint are as arch, tented arch, whorl, ulnar and radial loop, the Fig. 2 shows the different types of whorl patterns from fingerprint design. A whorl is portrayed by two deltas and one focal roundabout center. The center may have various examples. It might be winding, concentric circles, vertically compacted circles or even of the state of eye of a peacock quill. The edges start from one end, rise and hover towards the 

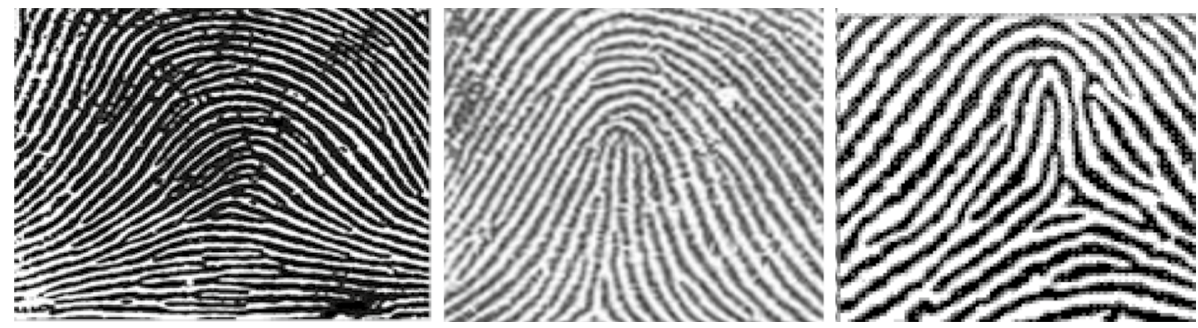

Fig. 1 Arch and Arch with Loop
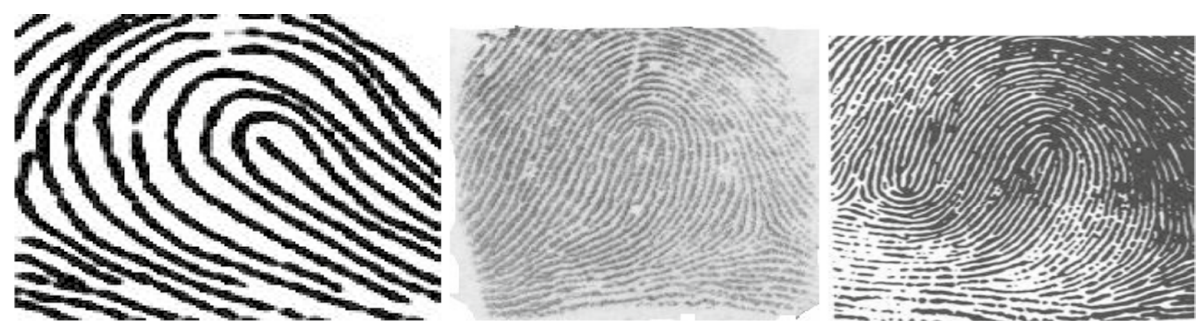

Fig. 2 Ulnar Loop, Radial Loop, and Composite Whorl

middle and go down towards the opposite end. Recent Advanced studies in genetics and developmental biology have guaranteed that the various projections of the human mind are physiologically associated with various fingers of both the hands. The practical coordination represented by the left half of the cerebral side of the equator is identified with the fingers of the right hand and the other way around. Consequently, the focalized left half of mind is associated with the fingers of right hand, the thumb is facilitated by the unrivaled frontal projection, index is associated with the mediocre frontal flap, middle finger with parietal projection, ring finger with the fleeting projection and little finger with the rear piece of cerebrum, which is the occipital flap. Correspondingly, the left half of the mind is associated with similar flaps of the cerebrum. Each projection zone is liable for a portion of the other impression of the encompassing (Singh and Majumdar 2015). Tented arch is a pattern that is portrayed by a straight upstanding edge at the center of a straightforward arch pattern. Loops are the most ordinarily up-to-the-minute highlights on a person's fingerprints just as in a subjective example space of a few fingerprints shown in Figs. 1 and 2. They are described by edges that start trickling out of a crosswise of the fingertip, circle from place to place the focal point of finger cushion, and back to a similar course where they began from. These loops can either flee from the thumb. Because of an individual area of arm bones-Radia and Ulna, any loop opening endlessly from thumb is an ulnar loop, and the one which opening near the thumb is a radial loop. A spiral whorl is described round patterns that are fit as a fiddle at the core or center. This pattern has two deltas at the two corners. The concentric whorl pattern is showed by having concentric rings of edge patterns. Lengthened whorl pattern is described by a long oval whorl flanked by two triradial on either side. Every single other component of a whorl is likewise present right now a pattern. It is one of the uncommon fingerprint patterns. It appears to be a Tai-Chi pattern at the inside or the center, encompassed by different roundabout layers of edges. Since the image has two symmetric yet oppositely situated arrangements, the subjects having imploding whorls grandstand doublemindedness. Composite whorl or twofold 

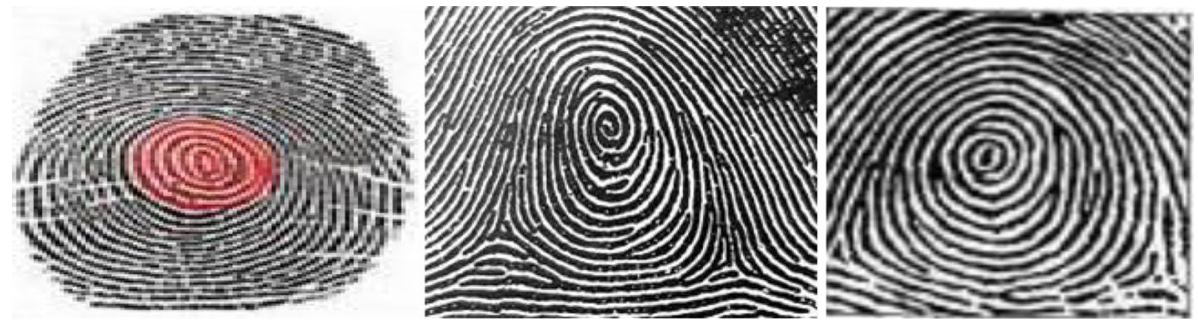

Fig. 3 Target/Concentric Whorl, Spiral Whorl and Concentric Whorl
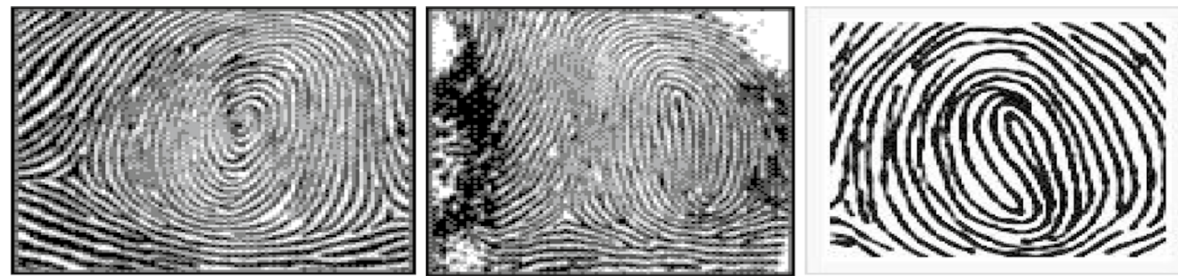

Fig. 4 Elongated Whorl, Imploding Whorl and Imploding Whorl

circle is one of the uncommon fingerprint patterns. It is either present on thumb or at most, the index finger. It is once more, one of the uncommon whorl patterns that contain a peacock's eye-molded circle contained inside a whorl. The center comprises of more than one spiral which are lined by a straight line at one of the corners. It to some degree seems as though the pattern on a peacock's tail quills. This pattern ought to have one triradius on either the left or right side. At the point when a pattern can't unmistakably have the option to sort into any of the above pattern types, comprises a blend of at least two above talked about patterns like a combination of concentric whorl and transformed loop and so on., it is professed to be a variation pattern. They don't contain any plain arch or loop, be outspread or ulnar. They do exclude any regular pattern. Although people have been utilizing fingerprints as a methods for recognizable proof for quite a while however right now, have put forth an attempt to make stride further to "study a connection between pattern of fingerprint and $\mathrm{ABO} \mathrm{Rh}$ blood group", with the goal that one can get a thought regarding the normal blood group from the investigation of finger impression pattern and the other way around. natural attributes like fingerprints and blood groups can't be overlooked and imitated like keys, passwords, and so on subsequently are viewed as increasingly dependable, true and solid in scientific sciences.

Other than an investigation of "blood group" commonness in itself isn't just significant for transfusion medication yet additionally for organ transplantation and hereditary research, forecast of specific malignancies/infections for certain blood groups just as in advancement contemplates that help researchers to comprehend the spot person involve in development's stretching tree (Fayrouz et al. 2011).

Different types of fingerprint patterns are as follows:

There are four unlike whorl patterns as: the plain whorl, the central pocket loop, the double loop, and the accidental whorl also it has different kinds of form shown in Figs. 3, 4 and 5. Their normal highlights are that they have at any rate two deltas and at least one of the ridgelines bends around the center to shape a circle or winding or other adjusted, continually bending structure. The accidental whorl can be any pattern 

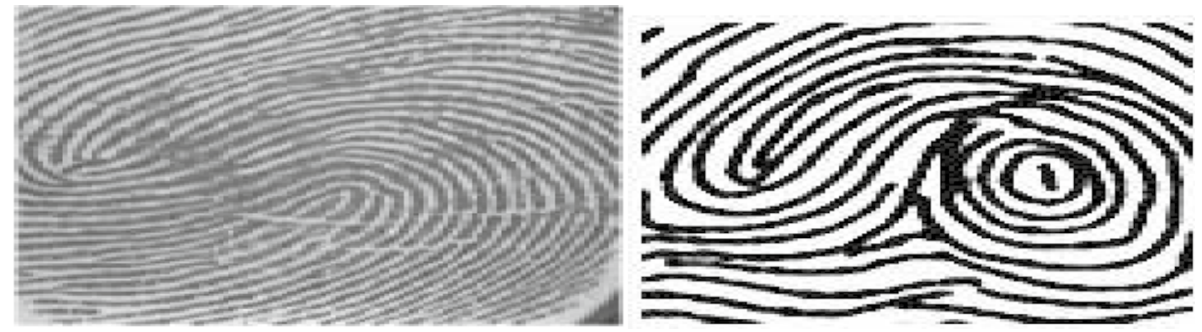

Fig. 5 Accidental Whorl or Variant and Accidental Whorl or Variant

or blend of patterns that don't fit into any of the above characterizations. The expression "Composite" is utilized to portray such patterns. Positive distinguishing proof utilizing fingerprints can be set up just if 16 to 20 purposes of closeness exist in the minutiae (Kanchan and Chattopadhyay 2006; Vij 2005; Subrahmanyam 1999). Arches are the most straightforward patterns and furthermore the rarest. There are two sorts: plain arches and tented arches. In these two types, the ridgelines stream into the print from one side, an ascent in the pattern, and stream out to the opposite side of the print. Loops are shaped by ridgelines that stream in from one side of the print, clear up in the middle like a tented arch, and afterward bend back around and stream out or will in general stream out as an afterthought from where they entered. Loops are assigned as being either spiral or ulnar, contingent upon which side of the finger the lines enter. The loop is the most well-known of the considerable number of patterns. Target/Concentric Whorl Spiral Whorl Concentric Whorl Elongated Whorl Imploding Whorl Imploding Whorl Accidental Whorl or Variant Accidental Whorl or Variant Most programmed frameworks for unique fingerprint examination depend on minutiae coordinating Minutiae are neighborhood discontinuities in the finger impression pattern. An aggregate of 150 distinctive minutiae types have been recognized some of the minutiae forms shown in Fig. 6. The ridge closure and ridge bifurcation minutiae types are employed as unique mark of acknowledgment.

Now a day's biometric applications are designed for
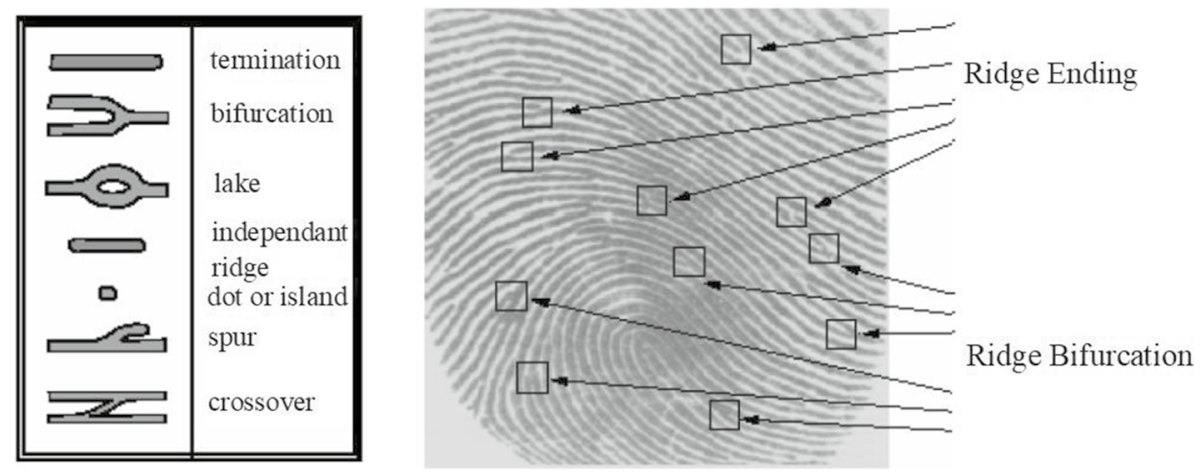

Fig. 6 Different minutiae types and Ridge ending \& Bifurcation (Fingerprint Identification - Project 2) 
- Authentication or Identification of an individual applications like e-records security, cellular phones access, medical records management, library access and virtual learning etc.

- E-Governance: like, Digital signature, Aadhar cards, Driver's licenses, border travel control, passport control, and welfare-disbursement etc.

- Digital Forensic: such as, corpse identification, criminal investigation, terrorist identification, parenthood determination, and missing children (Ramasubramanian and Alexander 2009).

Blood group structures were discovered way back in 1900 by Karl Landsteiner. Total 19 foremost groups have been identified which vary in their occurrence of spreading various races of mankind. Clinically, only 'ABO' and 'Rhesus' groups are of major importance. 'ABO' system is additional discriminated as A, B, AB, O blood group types according to presence of corresponding antigen in plasma (Ferraz 2013). Yet another biological record that remains unchanged throughout the lifetime of an individual is the blood group. Determining the blood group of a person from the samples obtained at the site of crime, helps identify a person. Landsteiner classified blood groups under the ABO blood group system (IMAQ 2004). Dermatoglyphics as a diagnostic aid used from ancient eras and now it is well established in number of diseases which have strong hereditary basis and is employed as a method for screening for abnormal anomalies. The study of possible predilection of certain disease and malignancies from blood groups are some of the factors which encourages one to carry the study further. Fingerprint minutiae patterns of ridges are determined as unique through the combination of genetic and environment factors. The identification of minutiae shown in Fig. 6, it shows minutiae like Ridge ending \& Bifurcation. Dermatoglyphics and its important role in the diagnosis of different diseases like hypertension, type- 2 diabetes and arthritis with genetic bases.

Machine learning is getting popular in all industries with the main purpose of improving revenue and decreasing costs; by using Machine learning technique they automate and optimize their process to solve challenging tasks very efficiently. The proposed research work aims in creating a system that finds the relationship between blood group and minutes patterns of fingerprints which will be helpful to predict blood group and common clinical diseases of an individual by analyzing its fingerprints. The fingerprint having basic four categories which are loop, whorl, arch and composites but also there are more than 100 interleaved ridge and valleys which explore unique characteristics of an individual which will help to design Deep Neural Network or Convolutional Neural Network (CNN) which predict blood group and common clinical diseases like hypertension, type 2-diabetes and arthritis. All ten fingerprints will be acquired in real time of both male and female from different age group and from various locations of country by using optical fingerprint scanner with external characteristics to from the large dataset as process. The fingerprint data will be acquiring using fingerprint scanner. All fingers of an individual are scanned to build dataset for training model with other necessary data collected by simple registration form where external attributes like age, weight, height, skin color, eyes color, work nature, eating habits (vegetarian or non-vegetarian), region (rural or urban), addiction (if any like drink, smoke), etc. will be recorded. The obtained fingerprint from database goes through various preprocessing stages for enhancement and removing the noise before feature extraction process which include Segmentation, Normalization, Orientation Estimation, Ridge Frequency Estimation, Gabor filter, Binarisation and Thinning from which the Orientation Estimation and Ridge Frequency Estimation. After the preprocessing of fingerprints, it goes through four steps of feature extraction, one is frequency domain feature 
vector obtaining by undergoing image through different levels of processing to build feature vector from acquired finger dataset. The all combined vectors within the dataset will then be allowed to pass for unsupervised training model. It uses clustering technique which helps to find similarity measure or relationship between features of fingerprint and external attributes. The clusters formed by unsupervised learning attempts to build neural network model which is deep neural network technique including features extracted from fingers and external attributes of an individual, it will be used to generate predictive model to know the blood group of an individual as well as it used to analyses disease arises with aging. The deep neural network model uses similarity measures or minimum distance for the entire combined feature vectors database. The proposed study divided into four different cases as Prediction of Blood Group, Analysis and Classification of Hypertension Disease, Analysis of Arthritis Disease and Analysis of Diabetics Disease.

\section{Literature review}

To work out the blood group of an individual, red cells of that individual are blended in with various neutralizer arrangements. If, for instance, the arrangement contains hostile to $\mathrm{B}$ antibodies and the individual has B antigens on cells, it will cluster together. On the off chance that the blood doesn't respond to any of the counter An or hostile to B antibodies, it is blood group O. A progression of tests with various kinds of antibodies can be utilized to distinguish blood group. On the off chance that the individual has a blood transfusion, the blood of the individual will be tried against an example of contributor cells that contains $\mathrm{ABO}$ and $\mathrm{RhD}$ antigens. If there is no response, contributor blood with a similar $\mathrm{ABO}$ and $\mathrm{RhD}$ type can be utilized. It shows that the blood has responded with certain antibody and is hence not perfect with blood containing that sort of counteracting agent If the blood doesn't agglutinate, it demonstrates that blood doesn't have antigens restricting the extraordinary immune response in the reagent. In the current framework, the blood group is resolved physically. Right now, arrangements, for solutions such as anti-a, anti-b, anti-d to the three samples of blood occurred. After some time, agglutination might happen.

Contingent on the agglutination, the blood group can be controlled by the individual physically. The weaknesses of this framework are more odds of human blunders are conceivable. Only specialists can tell the blood type by observing at the agglutination procedure. The traditional method of distinguishing the blood group is usually the plate test and the tube test (Pimenta et al. 2012). Both of which are performed by under comprehensive analog procedures with human observation. In the current era of digitization, it is not an efficient way to handle such a basic yet indispensable medical technique in a full analog atmosphere. There are also a few techniques such as micro plate testing and gel centrifugation (Pimenta et al. 2012; Ramasubramanian and Alexander 2009).

Fernandes et al. (2015) presented result in his research paper allow concluding that $\mathrm{ABO}, \mathrm{Rh}$ phenotype, reverse, and Crossmatching individuals blood group is possible with the developed device and procedure. They proposed device that allows blood type identification near the patient, outdoor a conventional laboratory, without the need of to be a specialized assistant to interpret the test result of blood, and in a very short time $(5 \mathrm{~min})$. The fast response time by device enables us it will be used in emergency situations, which is an advantage compared with the automatic commercial systems used in clinical laboratories (in average, response time of $30 \mathrm{~min}$ ). In addition, the methodology and test protocol applied to the sample's preparation is simple, without the need of sample dilutions or 
incubations. The prototype was implemented with noncomplex electronic components for a low-cost device. The implemented device distinguishes agglutinated from non-agglutinated samples using a classification algorithm (developed by the authors), based on the variation of OD discrete values of samples, for each blood test. The device operation was validated for $\mathrm{ABO}, \mathrm{Rh}$ phenotype, reverse, and Crossmatching human blood typing based on donor's blood samples provided by the IPST and test results agreed with their typing using their gold standard commercial and automatic systems.

Pimenta et al. (2012) The examination group is working in the advancement of programmed and scaled down gadgets for clinical applications. A case of this work is the advancement of a scaled down, minimal effort, versatile and programmed framework to blood typing in crisis circumstances, considering a spectrophotometric approach and within the sight of agglutination (cooperation between red blood cells' surface and explicit reagents). The use of a basic and quick exploratory convention permits deciding blood typing and empowers the structure of an electronic programmed framework. This framework will be helpful to decrease a few impediments of the current frameworks and techniques to blood typing. The outcomes can be influenced by a few variations that makes more enthusiastically the structure of a programmed framework, for example, the trial framework utilized for spectrophotometric estimations; the agglutination quality, which influence the contrasts among control and test samples; the time spent in test readiness since it is important blood and reagents weakening; and perform spectra estimation as quickly as time permits in light of the fact that the agglutinated cells continuously will in general settle in the base of the cuvette.

Proposed method by Fernandes et al. (2013) depends on the examination of the Rh phenotypes in human blood type dependent on the plate test and utilizing a spectrophotometric approach. This examination will be remembered for the versatile gadget recently created by the exploration group for deciding $\mathrm{ABO}$ human blood type. In this way, this paper presents the Rh phenotype assurance, including the D, C, c, E and e antigens, utilizing optical retention estimations, in the obvious range, to recognize an agglutinated test (cooperation among antigens and antibodies) from a non-agglutinated test (no association). To decide the nearness or nonappearance of every antigen five samples were set up by setting $50 \mathrm{f}$. $1 \mathrm{~L}$ of the particular reagent and 12.5 f.lL of entire blood in the plate, as depicted in the reagents manual. Every arrangement was blended for around one moment in a region of 2.5 $\mathrm{cm} 2$. At that point, the plate was situated in the estimating set-up of the spectrophotometer. An O.D. range estimation of the $50 \mathrm{f}$.lL reagents was likewise important to set the pattern and to additionally adjust the necessary gadgets.

The authors Pimenta et al. (2012) presents the standards for the improvement of a scaled down, ease, compact and programmed framework, in view of a spectrophotometric approach, are introduced. The framework will have the option to decide ABO and $\mathrm{Rh}$ blood types in a brief timeframe and in situ, which is reasonable to crisis circumstances and permit the blood typing outside an ordinary clinical research facility. For that, the essential components of the framework ought to be: a light source, a light receptor and a microcontroller. 1. Approval of the general test convention the convention applied in the framework use blood samples (from the Portuguese Blood Institute) and business antibodies as reagents (monoclonal Anti-An, Anti-B, Anti-AB and polyclonal Anti-D from Hos Lab Diagnostic). Four test samples should be set up for each blood test. Each test is acquired by blending blood in with a immune response. Blending blood in with the reagents it tends to be acquired two kinds of samples: agglutinated, if there is antigen-counter acting agent connection; or non-agglutinated if there is no association. For instance, blending A positive blood type with Anti-A, Anti-AB or Anti-D 
it is gotten an agglutinated test since this blood type has the A and D antigens. With the Anti-B, it is gotten from a non-agglutinated test. 2. Scaling down of the test framework After the approval of the spectrophotometry estimations to human blood typing, with the use of a quick and straightforward convention, the following stage was the execution of a particular light source framework by utilizing Light Emitting Diodes (LEDs) and a photodiode estimating gadget (S1336-5BQ photodiode from Hamamatsu), e.g., keeping away from the massive and costly framework dependent on a light source and monochromator.

Ramasubramanian and Alexander (2009) a coordinated fiberoptic a microfluidic gadget for the location of agglutination for blood type crossmatching has been portrayed. The gadget comprises of a straight microfluidic channel through with a responded RBC suspension is siphoned with the assistance of a syringe siphon. The stream meets an optical way made by a producer got fiber optic pair incorporated into the microfluidic gadget. A $650 \mathrm{~nm}$ laser diode is utilized as the light source and a silicon photodiode is utilized to recognize the light power. The separating between the tips of the two optic filaments can be balanced. At the point when fiber separating is enormous and the centralization of the suspension is high, the dispersing wonder turns into the prevailing system for agglutination identification while at low focuses and little dividing, opto-interruption turns into the predominant component. An agglutination quality factor (ASF) is determined from the information. Studies with an assortment of blood types demonstrate that the detecting technique effectively distinguishes the agglutination response in all cases. A dispensable coordinated gadget can be intended for future usage of the strategy for the close bedside pre-transfusion check. Stomach muscle positive blood type will respond with against A, hostile to B, and against $\mathrm{D}$ antibodies and cause agglutination. Henceforth, we just present outcomes for $\mathrm{AB}$ positive sort in detail right now. The information is illustrative of the outcomes acquired Mouad.

Ali et al. (2016) proposed fingerprint Recognition framework is separated into four phases. First is the Acquisition stage to catch the fingerprint picture, the second is the Preprocessing stage to enhancement, binarization, thinning fingerprint picture. The third stage is the Feature Extraction Stage to remove the element from the thinning picture by use minutiae extractor strategies to separate ridge ending and ridge bifurcation from thinning. The fourth stage is coordinating (Identification, Verification) to coordinate two minutiae focuses by utilizing the minutiae matcher technique in which closeness and distance measurements is utilized. The calculation is tried precisely and dependably by utilizing fingerprint pictures from various databases. The fingerprint acknowledgment framework is separated into three phases that are fingerprint picture pre-processing, include extraction and coordinating. The coordinating stage is partitioning into two procedure ID and confirmation. At the hour of catch the fingerprint picture, the pre-processing stage is applied to it. The yield of this stage will be passed to the component extraction organize which separates the minutiae point (ridge ending, Bifurcation) from thinning fingerprint picture, at that point the bogus minutiae evacuation is applied to remove genuine minutiae. At long last, the genuine minutiae are put away in tangle lab record. At that point if the fingerprint is as of now selecting? at that point send it to the coordinating stage in any case do the enrolment stage and store it in the database as a format. In ID case (one-to-many coordinating), the info include set, which is coordinating with $\mathrm{N}$ format from the database, $\mathrm{N}$ coordinating will be finished. The outcome will be considered as a coordinating Score. If coordinating Score more like 1, at that point the two fingers from a similar client. If coordinating score close to Zero, at that point the two fingers from deferent clients. In confirmation case (coordinated coordinating), the info includes set, which is coordinating with one layout from 


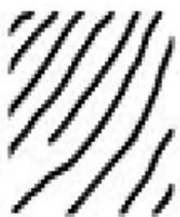

Ridge Ending

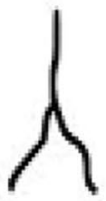

Bifurcation

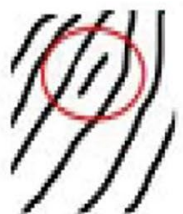

Short Ridge

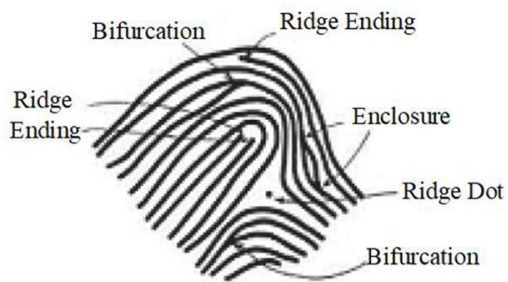

Fig. 7 Minutiae feature for different blood group fingerprint (Siva Sundhara Raja and Abinaya 2019)
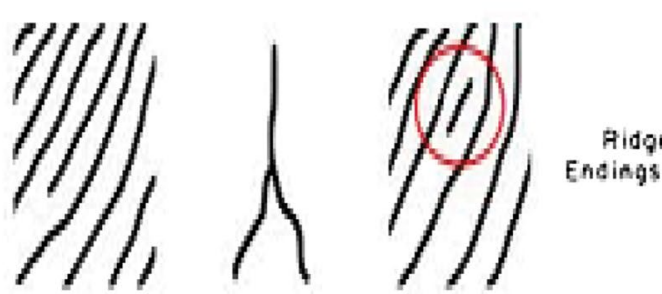

\section{Ridge Ending Bifurcation ShortRidge}

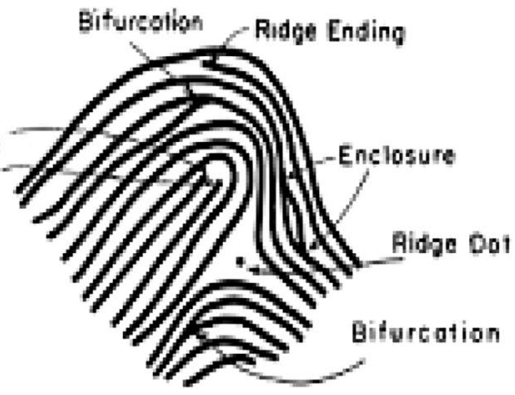

Fig. 8 Ridge, Terminations, valley, Bifurcations (Jain and Singh 2015)

the database, one coordinating will be done and chosen either the information fingerprint checked or unsubstantiated.

Siva Sundhara Raja and Abinaya (2019) proposed work comprises of the accompanying stages as pre-processing, feature extraction and classification, as portrayed in Fig. 7. The input pre-processing is the method to play out certain tasks for improving pictures preceding computational processing. It is a system that is utilized to conceal the data that isn't appropriate to the picture for additional processing. The pre-processing steps incorporate the accompanying: picture enhancement, picture resizing procedure, and thinning process. The picture enhancement process amends the lucidity of ridges and valley structure in the fingerprint picture. Right now, the histogram evening out technique is utilized. They have taken two types of blood gatherings. mage resizing is utilized to extend or compress the all outnumber of pixels. With the goal that it has the predetermined number of lines and sections. The focal point bending is done when we zoom the focal point, it will transform into a bent shape rather than the keener shape. Thinning is a morphological activity that is utilized to dispose of picking front picture components from double pictures. The feature extraction is a technique for catching the visual substance of pictures for ordering and recuperating. The methodologies depend on GLCM, Wavelet Features, Laws of surface features, Minutiae Extraction. The Major features of the fingerprints like ridge endings, ridge bifurcations are called Minutiae shown in Fig. 8. Minutiae states the distinction between one fingerprint from another fingerprint. A ridge ending is the place the ridge suddenly ends while ridge bifurcation is the place the ridge isolates into at least two branches. The extraction of minutiae turns out to be even more testing on account of the commotion present and lack of difference in the picture.

Ravindran et al. (2017) proposed work taken blood sample images and pre-processed it by using various techniques such as color plane extraction, color to gray image conversion. 
These blood image pre-processing terms can dramatically increase the uniformity of a visual investigation of collected samples. Also, they are applied several filter processes which strengthen or reduce certain image details enable an easier or faster assessment. Operators can augment a camera image with just a few clicks. Filtering encompasses several image filters for image optimization mixed filter for edge detection improvement, noise suppression, character alteration, etc. Image processing includes It includes several functions for image processing. Contrast increase by static or dynamic binarization, lookup tables or image plane separation. Resolution reduction via binning.

The methodology proposed by Rhiannon S. McBean et al. (2014) having two different genetic technologies: single nucleotide variant (SNV) mapping by DNA microarray and second method was massively parallel sequencing (MPS), concerning blood bunch genotyping. The steadiest transmissible change related with blood group bunch antigens are SNVs. To perform prediction of the blood type antigen phenotypes, SNV mapping which includes profoundly multiplexed genotyping can be performed on business microarray stages. Microarrays recognize just known SNVs, along these lines, to type uncommon or novel alleles not represented in the cluster, further Sanger sequencing of the district is frequently required to determine genotype. A model talked about right now the recognizable proof of uncommon and novel RHD alleles in the Australian populace. Enormously equal sequencing, otherwise called cutting edge sequencing, has a high throughput limit and maps all purposes of variety from a reference grouping, taking into consideration recognizable proof of novel SNVs. Instances of the use of this innovation to determine the hereditary premise of vagrant blood bunch antigens are presented here. In general, the assurance of a full profile of blood bunch SNVs, notwithstanding serological phenotyping, gives a premise to the arrangement of perfect blood in this manner offering improved transfusion security.

Ferraz et al. (2010) has developed technique that allows to analyses an image captured by a CCD camera detecting the occurrence of agglutination, through image processing techniques developed for determine the occurrence of agglutination. Secondly allows determine the blood type of the patient through the classification algorithm developed. Finally, allows store the information in a database built. The built database can store images captured and used in image processing techniques (each image contain four samples of blood and reagent), the standard deviation calculated in each four samples of the image, the result based by the value of standard deviation obtained for each of the samples (if agglutinated or not agglutinated in the sample of blood and reagent) and the result obtained by the classification algorithm (corresponding of blood type). The image will be processed by image processing techniques developed with the IMAQ Vision software from National Instruments (IMAQ 2004). The descriptions of all the functions presented are presented in the references mentioned (IMAQ 2004; Relf 2003).

The strategy proposed in Tejaswini and Mallikarjuna Swamy (2014) caught pictures of slide tests were a camera comprises of a shading picture made from three examples of blood and reagent. The picture preparing technique is probed the few pictures gained. These pictures are prepared utilizing MATLAB programming. The picture preparing strategies, for example, shading plane extraction, thresholding, and morphological activities were performed on the pictures. The picture got in the wake of applying auto thresholding grouping capacity it very well may be seen that the item and foundation are isolated. In the following stage, neighborhood limit activity utilizing Niblack work is applied it ascertains a pixel-wise edge and it very well may be seen just the outskirt portioned picture. The Narkis Banu and Kalpana (2018) and Relf (2003) present the blood group identification using blood cell images which takes from slide tests. Picture acquired by the utilization 
of cutting-edge morphology; it very well may be seen that the portioned picture is filled utilizing shutting activity. Progressed morphological activity Opening is performed it tends to be seen that it smoothens the shapes of cells by evacuating little articles. At that point the pictures acquired by applying the shading plane extraction HSL luminance plane and measure work. At long last, the blood gathering can be resolved. The utilization of picture preparing procedures empowers programmed discovery of agglutination and decides the blood sort of the patient in a short interim of time. The strategy is appropriate and accommodating in crisis circumstances.

Keerthana and Ranganathan (2017) build up an inserted framework that utilizes an Image preparing calculation to perform blood tests dependent on blood composing frameworks. Along these lines, the framework permits us to decide the blood sort of an individual killing customary transfusions dependent on the rule of the all-inclusive contributor, decreasing transfusion response dangers and capacity of result without human mistakes. This paper helps in lessening human intercession and perform total test independently from adding antigens to definite age of the outcome and gives the outcomes in most limited conceivable term with exactness and precision alongside capacity of result for additional references. Actualizing a quality framework in the lab limits mistakes and guarantees that the correct test is performed on the correct example, the correct outcomes got, and the correct blood item gave to the correct patient at the ideal time. The proposed framework presents the plan and usage of a smart compact gadget that gives the best possible data that we require for the investigation with the decreased expense and the profoundly prepared administrators are not required. This framework utilizes an AI calculation like a neural system that underpins MATLAB programming for blood bunch recognizable proof and identification of blood check examination. This framework additionally discovers an answer utilizing various calculations and strategies which gives us the most extreme precision in blood bunch distinguishing proof and tallying.

The work proposed by Berlitz et al. (2012) utilized protein A covering of the gold surface of QCM biosensors for the immobilization of antibodies against blood bunch antigens $A n$ and $B$, which allows the recognizable proof of the four principle blood bunches $\mathrm{A}, \mathrm{B}, \mathrm{AB}$ and 0 with two estimations. The Rhesus framework was inspected with various examinations on and the fruitful recognition of Rh-D, Rh-C, Rh-c, Rh-E and Rh-e antigens on human erythrocytes. The brisk, simple and dependable identification of blood bunch antigens $\mathrm{An}$ and $\mathrm{B}$ offers the chance of deciding the patient's association to the AB0 blood bunch framework by two estimations on hostile to An and against B sharpened quartz sensors. In Satoh and Itoh (2004) author also proposed a method which uses genetic analyzer for blood group prediction is recognized by the presence of three common representative alleles such as A, B, and O, in Satoh et al. (2001) the author proposed the analysis of four SNPs at nucleotide positions 261, 796, 802 and 803 to reflect serologic specificity. Sensor cells organized in equal and working on weakened entire blood without earlier planning of the blood tests will lessen the necessary time to approx. $3 \mathrm{~min}$. Swapping the quartz sensors, encouraged by the card mounted sensors and the licensed module holder, broadens the blood bunch investigation into the Rhesus, Kell and further blood bunch frameworks. In any event, for blood bunch antigens with a low antigen number for every cell, techniques are accessible to get ready biosensor coatings with satisfactorily upgraded affectability.

The proposed method utilized by Dalvi and Kumar Pulipaka (2018) three samples of blood are blended in with three distinct reagents namely anti-An, anti-B and anti-D is taken on a slide. After some time, agglutination may or may not occur. After the occurrence of agglutination, the slide containing three samples of blood blended in with three distinct reagents is captured as an image and allowed to process in MATLAB image processing 
toolbox. This framework lessens the chances of false detection of a blood group. Image processing techniques utilized for blood group detection are 1. Pre-processing techniques, 2. Thresholding, 3. Morphological operations, 4. HSL plane 5. Quantification. The color plane contains color information in image s. 'Comparing' sections in an image is the concept utilized in image processing. Comparison in Grayscale involves straightforward scalar algebraic operators. In color plane extraction, they first convert the RGB image into a gray image and then channel the obtained outcome utilizing median separating. Thresholding operation in image processing is utilized to create binary images. The grayscale samples are grouped into two parts as background and object. Right now, thresholding is performed utilizing Otsu's method. More than one threshold is resolved for a given image and segmentation is done creating certain regions. One background with many objects is the consequence of this staggered thresholding. It is a bunching based image thresholding. Morphological is a tool for extraction image components that are valuable in the representation. In morphological operation, there are two fundamental operations, for example, dilation and erosion as far as the union of an image with a translated shape called an organizing component. Here, closing operation is performed where dilation is followed by erosion. Also, edge detection utilizing the Canny edge detection strategy is performed. Morphological operations are utilized to eliminate noise spikes and ragged edges. HSL plane stands for Hue, Saturation, and Luminance. It is the representation of the RGB color model. Shade is expressed in a degree around a color wheel, while saturation and brightness are set as a percentage. Quantification is expressed as a number or measure of quantity. It measures power only in the region of the intrigued area. Area (percentage of surface examined for full image), mean (average value of the pixel), standard deviation, least and maximum values of pixel power are resolved. Also, region properties are extracted. Utilizing the value of standard deviation, the occurrence of agglutination is recognized and accordingly the blood group is resolved.

Fayrouz et al. (2011) study reveals an association between the pattern of the unique mark and $\mathrm{ABO}$ blood group. With ongoing advances in unique mark detecting technology and improvement in the accuracy and matching velocity of the finger impression matching algorithms, automatic personal identification is becoming an attractive/complement to the traditional methods of identification. As biometric technology matures, there will be an increased interaction among the biometric market and its identification application, since fingerprints will remain an integral part of the preferred biometric-based identification solutions in the years to come, a relationship of unique finger impression pattern to blood group presents scope for additional identification data which can be utilized for personal identification purpose, also investigation of possible predilection of certain disease and malignancies from blood groups are some of the factors which encourages one to carry the examination further. Chosen randomly having distinctive ABO blood groups, with the objective to a) Study the distribution of unique finger impression pattern among the subjects having diverse $\mathrm{ABO}$ and $\mathrm{Rh}$ blood group $\mathrm{b}$ ) Correlate any relation between their characters and blood group. The data from the investigation showed that the male: female ratio was 1.2:1. Most subjects (48.9\%) right now of blood group $\mathrm{O}$ followed by blood group $\mathrm{A}$ (33.1\%), B (12.8\%) and $\mathrm{AB}(5.2 \%)$. Rh-positive cases constitute about $87.2 \%$ of all considered cases. The general distribution of the pattern of finger showed a high recurrence of Loops enlisting 50.5\%; followed by whorls (35.1\%) and arches (14.4\%). In Rhpve cases of blood group An and O loops occurrences were the most elevated (52\% and $4.3 \%$ individually) at that point whorls (33.4\% and 30.6\% separately), while in blood group B whorls were predominance in both Rhpve and Rh_ve cases. In all blood groups, there was the high recurrence of loops in thumb, record and little fingers. 
Ferraz (2013) utilizes the slide test and image processing techniques utilizing the IMAQ Vision from National Instruments. The image captured after the slide test is processed and recognizes the occurrence of agglutination. Next, the classification algorithm decides the blood type in the analysis. Finally, all the information is stored in a database. In this way, the framework allows deciding the blood type in a crisis, eliminating transfusions based on the rule of universal donor and diminishing transfusion reaction dangers. This framework is based on a slide test for deciding blood types and the software developed utilizing image processing techniques. The slide test consists of the blend of one drop of blood and one drop of each reagent, anti-An, anti-B, anti-AB, and anti-D, being the outcome interpreted according to the occurrence or not of agglutination. The agglutination reaction means that occurred reaction between the antibody and the antigen, indicating the presence of the antigen appropriate. The combination of the occurrence of agglutination, or nonoccurrence, decides the blood kind of the patient (Datasheet of DiamedDiaclon Anti-A 2008; Fingerprint Identification - Project 2). Accordingly, the software developed based in image processing techniques allows, through an image captured after the procedure of the slide test distinguish the occurrence of agglutination and consequently the blood sort of the patient.

Thakar and Sharma (2016) process remarkable highlights which they found inside the fingerprint designs which make us equipped for offering input. In any case, different examinations have demonstrated that even the prepared and experienced specialists submit different sorts the fingerprint, these might be a result of the utilization of discretionary/nonstandard phrasing like clockwise/anticlockwise or bearings and so forth recorded as a hard copy a report. The traditional technique for fingerprint correlation with the focal point to find details in bearings, which is a tedious system should be changed. Right now, a framework, optical sensor-based per users are utilized to peruse and gained fingerprint pictures in the accompanying three phases: Firstly, picture handling calculations are utilized to get dark tone impressions of the fingerprint picture. Also, the prepared picture is accordingly used to extricate the details (just bifurcations and ridge finishing). The third step is examining the position of various details (bifurcations and ridge finishing) on fingerprints, with the assistance of situation coordinated calculations. The separated pictures are contrasted, and the databases present in the framework and results are gotten. In any case, as opposed to the abovementioned, in the present examination, a semi-self-enough method has been utilized. In this way, in the present examination, a far-reaching endeavor has been made to extricate physically all the particulars present in the unique mark with the assistance of Adobe Photoshop (CS5) Software and to build up an adjusted lattice which can be utilized to methodically discover the position of the details alongside estimating certain extra element like Angle.

Azhagiri et al. (2018) study uncovers a huge relationship of blood groups O, A, B, AB to Hypertension, Peptic ulcer, Anemia, Rheumatoid Arthritis, Gastritis, Diabetes, and Bronchial asthma. The transcendence of the circle was most noteworthy among all blood groups. As indicated by this investigation, following outcomes were watched, Loops were the most well-known unique finger impression example and Arches were the least normal, Whorls and blended were moderate, Highest quantities of Loops were found in blood groups $\mathrm{O}, \mathrm{B}$ contrasted with $\mathrm{An}$ and $\mathrm{AB}$, Blood bunch $\mathrm{O}$ positive is the most well-known, $\mathrm{O}$ negative and $\mathrm{AB}$ negative is the rarest, Loops, whorls, blended and arches were most elevated in females, Group A was the most widely recognized blood bunch among guys, Blood bunch O, B, were the most regularly observed blood groups in females, Some basic clinical grumblings were found in all the blood groups.

Kanchan and Chattopadhyay (2006) the result shows that each fingerprint is exceptional; loops are the most normally happening fingerprint design while arches are the least 
normal. Guys have a higher occurrence of whorls and females have a higher frequency of loops. Loops are transcendent in blood groups A, B, AB and $\mathrm{O}$ in both Rh-positive and Rhnegative people except for in $\mathrm{O}$ pessimistic where whorls are progressively normal. They can reason that there is a relationship between the conveyance of fingerprint designs, blood gathering, and sexual orientation and along these lines expectation of sex and blood gathering of an individual is conceivable dependent on his fingerprint design.

Rastogi and Pillai (2010) present investigation shows that there is an association between the appropriation of fingerprint designs, blood gathering and sexual orientation. The discoveries of the examination can be finished up as follows: Each fingerprint is one of a kind consequently it tends to be successfully utilized as a proof for distinguishing proof in the courtroom. Loops are the most generally happening unique mark example and Arches are the least normal. Blood bunch $\mathrm{O}$ positive is the most widely recognized and A negative is the rarest. Loops are overwhelming in blood bunch $\mathrm{A}, \mathrm{B}, \mathrm{AB}$ and $\mathrm{O}$ in both $\mathrm{Rh}$ positive and $\mathrm{Rh}$-negative people except for in $\mathrm{O}$ adverse where whorls are progressively normal. Whorls are progressively normal in blood bunch $\mathrm{O}$ negative. Loops and arches are most extreme found in blood bunch some time whorls are increasingly regular in blood bunch O. Blood groups An and B were the most widely recognized (similarly dominating) among guys, blood bunch $\mathrm{O}$ was the most generally observed blood bunch in females. Guys have a higher frequency of whorls and females have a higher rate of loops. Hence prediction of sexual orientation and blood gathering of a for every child is conceivable dependent on his fingerprint design. Comparative examinations ought to be led to a bigger example in order to build the precision of prediction.

Joshi et al. (2016) present examination uncovers that there was an association between dissemination of fingerprint (dermatoglyphic) design, Gender and blood groups. The general circulation example of the essential fingerprint was of a similar request in people with ABO; Rh blood groups for example high recurrence of loops, moderate of whorls and low of arches. The discoveries of the examination can be finished up as the Loops are the most normally happening unique mark example and Arches are the least normal, Blood bunch $\mathrm{O}$ positive is the most well-known and A negative is the rares, Loops are prevalent in blood bunch $\mathrm{A}, \mathrm{B}, \mathrm{AB}$ and $\mathrm{O}$ in both $\mathrm{Rh}$ positive and $\mathrm{Rh}$ negative people aside from in $\mathrm{O}$ antagonistic where whorls are increasingly normal and Males have a higher occurrence of whorls and females have a higher frequency of loops.

Jha et al. (2015) study uncovered that blood bunch AB and O had a most noteworthy rate of loops (76.92\% and $71.05 \%$ separately) trailed by whorls $(23.08 \%$ and $28.95 \%$ individually), comparatively in blood bunch An and B the design of the loop was normal with $57.14 \%$ in the two cases. The study inferred that there is a solid connection between blood groups and fingerprint design. From the examination, it was presumed that the recurrence appropriation loops design were most noteworthy in blood bunch $\mathrm{AB}$ (76.92), $\mathrm{O}$ (71.05\%) and B (57.14\%) individually. So also, the examination likewise inferred that the dispersion of whorls was most elevated in blood bunch A with $42.86 \%$ circulation, then in blood bunch B with 39.28\% dissemination and for blood bunch AB it was discovered $23.08 \%$ and arches were least in blood bunch B with $3.70 \%$ appropriation. Further investigation ought to be completed by expanding the example size to get the progressively exact portrayal of the populace and need increasingly comparative examinations in different districts as well with the goal that near examination should be possible.

The study proposed by Sudikshya et al. (2018) corresponds to the connection between different patterns of fingerprints and "ABO" blood groups and "Rh" blood types in Nepalese guys and females. In spite of the fact that they realize that fingerprints are rarely indistinguishable and they never show signs of change from birth till death, 
this examination is an endeavor made to connect fingerprints with sex, diverse blood groups, and $\mathrm{Rh}$ blood types which may thus improve the legitimacy of fingerprints in distinguishing proof and legal medication and can be utilized for conceivable prediction of specific illnesses. From the present investigation, the accompanying ends are drawn: (1) Loops are the most regularly discovered fingerprint pattern and arches are minimal normal in the two guys and females and furthermore in "ABO" blood groups. (2) The recurrence of loops is most noteworthy followed by whorls and arches in Rh +ve blood types, while the frequency of whorls is most elevated followed by loops and arches in $\mathrm{Rh}$-ve blood types. (3) Our outcomes uncover the most elevated rate of loops in the center and little finger in all blood groups, while the whorls are usually found in ring fingers in all blood groups. The frequencies of whorls are likewise most elevated in forefinger and thumb in all blood groups aside from in blood bunch "O" where loops are as often as possible present. (4) From this investigation, they can infer that circulation of essential pattern of the fingerprint isn't identified with sexual orientation and $\mathrm{ABO}$ and $\mathrm{Rh}$ blood gathering, yet its conveyance is identified with singular digits of two hands.

Narayana et al. (2016) they present the examination is an endeavor to relate fingerprint patterns with sexual orientation and blood gathering of a person. Fingerprint patterns can be of help in anticipating the sexual orientation and blood gathering of a person. It might help in expanding the legitimacy of fingerprints in recognizable proof of people and tackling of wrongdoings. They got results areas: Loops were the most generally discovered pattern and composite the least. In the loop pattern, the commonest pattern was an ulnar loop, which was measurably huge right now. Blood bunch $\mathrm{O}$ positive was the most wellknown and $\mathrm{AB}$ negative was the rarest. Rh-positive blood groups were more contrasted with Rh-negative blood groups, which is demonstrated right now and critically dependent on the information too. Blood bunch B was the most widely recognized among Rh-positive blood groups followed by $\mathrm{O}$, An and AB blood groups. Among Rh-negative B and A blood groups were similarly predominant followed by $\mathrm{O}$ and $\mathrm{AB}$. Loops were most elevated in guys, whorls and arches were most noteworthy in females. Loops were prevalent in all the blood groups with the exception of A positive where whorls were prevailing. The most elevated number of the considerable number of patterns was found in blood bunch $\mathrm{O}$ and least in $\mathrm{AB}$ among Rh-positive blood groups and factually demonstrated critical right now. Composites were least generally found in all the blood groups.

The Study by Sangam et al. (2011) they found the loops were connected more with $\mathrm{O}$ gathering, whorls with $\mathrm{AB}$ gathering and arches with $\mathrm{B}$ gathering. Thumbs introduced a high recurrence of whorls in $\mathrm{A}+$ ves. Record and ring fingers were related to the high recurrence of whorls in A-ves and $\mathrm{AB}+$ ves. So the prediction of blood gathering somewhat might be conceivable with the investigation of unique finger impression patterns which might be of extraordinary incentive in criminological medication, however, impact provincial varieties, sex and hereditary variables ought not to be disregarded.

Deopa et al. (2014) has an endeavor has been made in the present work to examine their connection with sexual orientation and blood gathering of a person. Loops were the most widely recognized (58.29\%) fingerprint pattern while whorls were moderate $(37.00 \%)$ and arches were the least normal (4.71\%). Guys had a higher rate of whorls and females had a higher frequency of loops. Loops are overwhelming in blood bunch $\mathrm{A}, \mathrm{B}, \mathrm{AB}$ and $\mathrm{O}$ in both Rh-positive and Rh-negative people aside from in 'A' constructive blood bunch where whorls prevail marginally. Whorls were most noteworthy in An and AB positive blood gathering, and loops were most elevated in $\mathrm{O}$ and $\mathrm{B}$ blood gathering. Arches were least in all blood groups. There is an association between conveyance of fingerprint patterns, blood 
gathering, and sexual orientation and in this way prediction of sex and blood gathering of an individual is conceivable dependent on his fingerprint pattern.

Shivhare et al. (2017) does the investigation which uncovered the association between dermatoglyphic, blood gathering and sex: Most subjects have a place with Rh-positive and $\mathrm{O}$ blood gathering. Loops are the regular and arches are extraordinary fingerprints. Loops were most noteworthy in $\mathrm{B}$ blood gathering and least in $\mathrm{AB}$ blood gathering. Whorls most elevated in An and least in B blood gathering. Arches were most elevated in AB and least in B. Loops higher in female and most reduced in male, whorls most noteworthy in male and least in female and arches most elevated in male and most reduced in the female. Loops were most noteworthy in Rh-positive and least in Rh-negative. Whorls most elevated in Rh-negative and least in Rh-positive. Arches were most noteworthy in Rh-positive and least in Rh-negative.

The investigation does by Radhika (2016) uncovered that there is an association between the dissemination of fingerprint patterns, blood gathering, and sexual orientation. Loop was most as often as possible seen fingerprint followed by whorl curve and composite. $\mathrm{O}$ positive is the most regular blood gathering and $\mathrm{AB}$ negative is missing. Loops are dominating in blood bunch $\mathrm{O}$ followed by $\mathrm{B}$ and $\mathrm{An}$ in Rh-positive subjects, trailed by whorls. Curve and composite were basic among $\mathrm{O}$ and A positive subjects.

Morris et al. (2016) proposed method having outcomes that fingerprint asymmetry could be formed into a significant instrument for anticipating the danger of Type 2 diabetes mellitus and Type 1 diabetes mellitus and that wavelet examination is a technique that can be utilized to evaluate asymmetry in fingerprints. The benefit of fingerprints scored utilizing wavelet-based strategies over hereditary testing, is that it can demonstrate gestational condition and would be considerably less costly. The expense is significant, given late reports that both hazard mindful and chance unconscious people were keen on hereditary testing, however distinguished the requirement for minimal effort tests. They propose an increasingly far-reaching examination of fingerprint asymmetry as a predictor of both T2DM and T1DM chance, scoring asymmetry with wavelet investigation and contrasting with the prescient capacity of hereditary qualities alone, is justified.

The propose work by used information digging Veena Vijayan and Anjali (2015) which can be used for evaluating distinctive disease patterns, remedial data extraction, quiet support and organization and finding of clinical parameters. Here a choice emotionally supportive network is recommended that predicts diabetes which utilizes choice stump as a base classifier in AdaBoost calculation. The framework utilizes a worldwide dataset taken from UCI vault of AI for preparing which contains 768 cases and 9 characteristics and utilizations nearby dataset for approval which had been gathered from better places in Kerala. The PC data framework with the AdaBoo-choice stump classifier gives a precision of $80.729 \%$ for anticipating diabetes with an extremely low estimation of blunder rate.

Tafa and Pervetica (2015) study the dataset which comprises of 402 examples taken from three unique areas in Kosovo. The characteristics of the database are BMI (weight file), glucose level before dinner and after the feast, the systolic and diastolic blood pressure, the genetic factor, the customary eating regimen, and day by day physical exercises. The last two qualities are assessed as follows. With respect to the issue of customary eating routine, while depending on contributions from the clinical clinicians, patients were inquired as to whether they took their dinners in roughly the same equidistant everyday interims, in any event, three times each day and furthermore if their suppers were not voluminous. The introduced approach depends on the joint usage of two calculations in Matlab that have been executed on the recently gained dataset with the various credits when contrasted with the past work right now. The calculations are executed and assessed freely 
however the dynamic depends on the joint results from the two calculations. The point of this methodology is to settle on the choice progressively dependable.

Mehta and Mehta (2015) study the one hundred type II diabetes mellitus patients (50male and 50 female) were chosen for study and contrasted and equivalent number of controls. Fingerprints were acquired by a printing technique. Parameters contemplated were arches, whorls, loops. Circulation of fingertip patterns indicated a noteworthy contrast between diabetics and controls. The appropriation of fingertip patterns on both ways submit male diabetics and controls. The whorls were altogether expanded though loops and arches were essentially diminished in male diabetics when contrasted with controls. The whorls were fundamentally expanded while loops were essentially diminished in female diabetics when contrasted with female controls. Nonetheless, arches were fundamentally diminished in the left hand of female diabetics.

Ameer et al. (2014) investigation right now spellbinding. An aggregate of one hundred patients partook right now was a completely known instance of diabetes mellitus. Out of these one hundred patients, most of the patients were having a place with a whorl pattern of fingerprints I-e. fifty half while the number of patients having a place with the Loop pattern was forty-five $45 \%$, the composite was just $2,2 \%$, and no specific patient having a place with Arch pattern. There is having to build up a definite and tremendous examination to investigate the association of fingerprint pattern with Diabetic disease. This investigation offered reasonable weighting on the circulation of fingerprint pattern among diabetic disease patients. Confinements of study where it was just restricted to clinical OPD patients and Limited uniquely to diabetes mellitus patients.

The investigation by Roshani et al. (2016) concluded WHORLS are the most regular pattern in both right and left hands of both male and female diabetic subjects and LOOPS are the most normal pattern in both right and left hands, in the two guys and females in non-diabetic subjects. The Arches were fundamentally diminished in both the right and left hand of males and females in diabetics and non-diabetic subjects. This examination shows a huge association between fingerprint patterns and diabetes in both genders. Study might be helpful to distinguish the high-chance people in the populace, for type- 2 diabetes mellitus; the most punctual prediction and finding of patients with type-2 diabetes mellitus will improve the aftereffect of treatment and further entanglements. Notwithstanding; there are a few investigations that appeared inverse outcomes to our examination; subsequently, there is having to do additionally contemplates and bigger examples ought to be analyzed in detail to additionally approve the discoveries of this examination and arrive at a complete resolution.

Smail et al. (2019) examination demonstrated that the type of loop in typical guys is $37.50 \%$, even with the diabetes female gathering the type of loop is $18.75 \%$. This investigation demonstrated that the control male arches bunch is no worth which is zero, while in the patient female the arches bunch esteem is high to $25 \%$. Confinements of the examination gave us that the control male whorls run is $6.25 \%$, however for the female patients is $12 \% .50$ which expanded worth. Their investigation demonstrated that the type of loop in typical females is $31.25 \%$, yet in the diabetes male gathering, the type of loop is $0 \%$. This investigation indicated that the control female arches bunch is $6.25 \%$, yet in the patient's male, the arches bunch esteem is high to $25 \%$. Another impediment of the examination gave us that the control female whorls run and the diabetes male or equivalent worth which is about $18.75 \%$. For the all-out typical and diabetes guys, the loop bunch in the ordinary male is higher than diabetes $(68.75 \%>18.75 \%)$. For the all-out ordinary and diabetes male the arches bunch in the typical male is lower than diabetes $(6.25 \%<50 \%)$. For the allout typical and diabetes male, the entire gathering in the ordinary male is underestimating 
than diabetes $(25 \%<31.25 \%)$. Arches are found in five percent of fingerprint patterns. The ridges run starting with one side then onto the next of patterns, making no retrogressive turns. Usually, there is no information in a curve pattern.

Igbigbi et al. (2001) have inspected the plantar and advanced prints of the sole of 99 indigenous Malawian patients matured 25-66 years going to the clinical outpatient center for diabetes mellitus, fundamental hypertension and a mix of the two conditions at Lilongwe and Queen Elizabeth focal emergency clinics. The gathering comprised of 27 diabetics (15 guys, 12 females), 21 hypertensives ( 9 guys, 12 females) and 51 diabetics with hypertension ( 21 guys, 30 females). All patients were analyzed as Type 2 diabetics after the age of 20 years. Their outcomes indicated that soles of all patients had a greater number of loops than arches and a greater number of arches than whorls, which were limited to the distal zones. In hypertension, whorls were found in zones I, II and III though, in patients with diabetes and hypertension, the whorls were found in zones I, III and IV. In digits, the most prevalent ridge pattern was arches in all patients, trailed by loops and whorls were missing. In the principal digit, diabetic patients had no arches, yet ladies' hypertensives demonstrated arches. In patients with diabetes and hypertension, arches were available in both genders yet in men it was limited to the correct foot. Loops were discovered distinctly in the first digit in quite a while. The recurrence of loops was most noteworthy in diabetic patients, high in diabetics with hypertension and least in patients with hypertension alone.

Tarca and Tuluc (2005) have considered a complete number of 133 patients with Type 1Diabetes Mellitus, out of which 58(33 guys and 25 females) were youngsters and adolescents of ages somewhere in the range of 4 and 18 years. The disease showed in these cases between the age of 2 and 17 years. Among the female patients, the loop was found in around equivalent extents in both the hands $(58.95 \%$ on the left hand and $60.60 \%$ on the correct hand) while if there should arise an occurrence of typical subject the loops were found on the left side as it were. The loop dissemination on the five fingers indicated an expanded recurrence on the fingers from V and III. Whorls were increasingly visited in the male arrangement and on the correct hands. Arches were increasingly visiting in the female arrangement. The presence of these markers, before the clinical sign of the disease, makes workable for their utilization in anticipation of insulin subordinate diabetes mellitus.

Udoaka and Lawyer-Egbe (2009) have considered an absolute number of 90(50 guys and 40 females) grown-up diabetic patients and the contrasted and the same number of ordinary subjects as controls. There was no huge contrast in the computerized patterns in the two groups. The atd point, dat edge, the absolute ridge include were fundamentally more noteworthy in the diabetic patients contrasted with the typical subjects. The pattern force record was higher in the diabetic guys however it was lower in the female diabetics. Their perceptions can be utilized for distinguishing proof of diabetics.

Nezhad and Shah (2010) have considered 30 patients of diabetes type 1 and 30 typical subjects as control gathering. The mean period of patients and control bunch was $22 \pm 11$ and $38 \pm 8$ individually. Among these $42 \%$ were guys and $58 \%$ were females. They have discovered that the state of loop and whorl are heterogeneous, and their number varies altogether contrast with a control gathering ( $p=0.001, p=0.004$.). The a-b 32 ridge includes demonstrated an expansion in the ridge considers as a real part of the diabetic men than control gathering. The atd point size in both the experimental group and control gathering of females was more than guys. These creators are of the sentiment that dermatoglyphics can be an appropriate strategy for hereditary examinations and diabetes type 1.

Sumathi and Desai (2007) have considered a sum of 100 patients of diabetes mellitus Type 2 and hypertension of either sex or age gathering of 35-55 years. They were 
coordinated with hundred controls. They discovered diminished a-b ridge include in female diabetics. The accompanying huge parameters have been found in their investigation in the palmar dermatoglyphics in type 2 diabetes with hypertension. In both male and female patients, there is the nearness of diminished I1 pattern and nearness of expanded I3 pattern in the left hand. The nearness of diminished whorls saw in two hands of male patients. The nearness of expanded ulnar loops and whorls in two hands of female patients.

Padmini et al. (2011) their exploration concentrate on dermatoglyphics in diabetes mellitus underlined that however dermatoglyphics by and large don't assume any significant job in clinical analysis yet, it can fill in as a pointer to pick out subjects from a huge gathering of individuals for additional examinations to affirm or preclude diabetes mellitus have studied fingerprints and palmar prints from 200 subjects, 100 guys and 100 females in the age gathering of 25 years to 80 years, of which $95 \%$ of cases were non-insulin subordinate diabetics and $5 \%$ of cases were insulin subordinate diabetics contrasted and 200 controls. Higher occurrence of variety in methods for ulnar loops (83.2), composite whorl (1.8), all-out finger ridge tally 33 (108.6), total finger ridge check (138.55), dat points of the right hand (59.77) and left hand (62.3) in diabetics than in controls was seen by them. The rest of the parameters were low in diabetics than in controls. In male diabetics increment in methods for ulnar loops (41.6), outspread loops (1.7), complete finger ridge check (106.25), supreme finger ridge tally (137.58), atd edge of right hand (41.68) and left hand (41.67), dat edges of left hand (62.42) and adt of right hand (80.45) was seen than in controls. In female diabetics, huge increment in basic arches (5.7), all-out finger ridge tally (110.94), total finger ridge tally (139.52), dat points of two hands right (61.6) and left hand (62.17) was seen than in controls.

Sharma and Sharma (2012) their investigation of 50 diabetic cases and 50 controls chose from the SMS Hospital, Jaipur, India, found that the complete finger ridge check, total finger ridge tally, and the a-b ridge include were higher in all the patients. The atd points in the hands of the two sides in the patients were expanded in all the groups aside from left side in guys. In any case, they varied fundamentally on the correct side and on the left side in females, $p<.001$. In the general groups right tda edge was huge. The smidgen and the tda edges on the two sides of the hands in all the groups were lower in the patients aside from left tda edge in 34 guys. Be that as it may, they varied just all together in the left bit, right tda in females. The aftereffects of their examination work demonstrated that dermatoglyphic variations from the norm might be utilized as an indicative instrument for anticipating the chance of the advancement of diabetes sometime in the future.

Taiwo and Adebanjo (2012) have completed an examination to explain whether fingerprint pattern of dermatoglyphics is related to withtype 2 diabetes or not. Dermatoglyphic information was acquired from controls and type 2 diabetic subjects going to the Diabetic Clinic of Lagos University Teaching Hospital. They saw all-out finger ridge tally was fundamentally higher $(p<0.05)$ in diabetic subjects than in nondiabetics. Considering the association between fingerprint pattern and type 2 diabetes, dermatoglyphics might be utilized for the early ID of hazard bunch people for reconnaissance purposes so as to forestall disease beginning.

Rakate and Zambare (2014) have looked at the distinctions in the complete finger ridge tally, a-b ridge includes and atd point in patients with type 2 diabetes mellitus with a control gathering. Their examination was completed on 75 type 2 diabetic patients (51 male and 24 female) of 30 to 60 years and 75 non-diabetic persons (47 guys and 28 females) of the equivalent 35 age bunch as a benchmark group. In their examination, they found an expansion in the number of whorls, absolute finger ridge tally, a-b ridge tally alongside more extensive atd point in type 2 diabetes mellitus patients. 
Desai and Hadimani (2013) have opined that dermatoglyphics is a developing order and its simple and prepared pertinence renders it as a valuable device to the clinician. The dermatoglyphics isn't to analyze and not for characterizing a current disease yet to forestall by anticipating a disease and to distinguish individuals with hereditary inclination to build up specific diseases. They have attempted to decide noteworthy palmar dermatoglyphic parameters if there should be an occurrence of sputum positive tuberculosis, diabetes mellitus type 2 with basic hypertension, dermatitis, innate coronary illness, and Down disorder and contrasted and the benchmark group. Their investigation demonstrated that there were some hereditary components that were engaged with the causation of different diseases referenced previously. Itis conceivable to anticipate from dermatoglyphics people's possibility of gaining disease. Noteworthy discoveries they watched were:1. The nearness of diminished whorls, 2. The nearness of expanded ulnar loops,3. The nearness of expanded simian line in the left hand of considered patients.

Shivaleela et al. (2013) have done an investigation to discover the recurrence of different fingerprint patterns in type 2 diabetes mellitus with and without ischemic coronary illness. Their examination likewise planned to discover the recurrence of fingerprint patterns in type 2diabetes Mellitus patients having the family ancestry of cardiovascular disease. Twenty-five type 2diabetes Mellitus male patients in the age gathering of 38-65 years were chosen, of which 18 had an ischemic coronary illness and 16 patients had the family ancestry of cardiovascular occasions. There was a higher recurrence of whorls in type 2diabetes Mellitus patients than different patterns. Less recurrence of arches, high recurrence of whorls and ulnar loops were seen in type 2diabetes Mellitus patients contrasted and type 2diabetes Mellitus patients without ischemic coronary illness. The thing that matters was not factually huge. Dermatoglyphics in type 2diabetes Mellitus and in patients with family ancestry of cardiovascular disease didn't show dominance of any of the fingerprints in ischemic coronary illness. Therefore, they opined dermatoglyphics might be symptomatic apparatus in type 2diabetes Mellitus however not in recognizing the hazard class for ischemic coronary illness.

Umana et al. (2013) completed an examination to decide the association between fingerprints patterns and diabetes mellitus utilizing subjects in Zaria, Nigeria. Their consequences of 101 type 2 diabetic patients were contrasted and 126 typical subjects. From their outcomes, there was an association between fingerprint patterns of guys with diabetes mellitus. From the above investigation, they reasoned that the male with a Arch pattern of fingerprint in their correct hand is inclined to create diabetes mellitus at a later stage.

Mittal and Lala (2013) have endeavored to discover an association of the dermatoglyphics patterns of the sound people and diabetes mellitus patients. An aggregate of 200 subjects took part in their investigation of which 100 were diabetic patients (50 guys and 50 females) and 100 were solid people utilized as controls (50 guys and 50 females). They signify 'atd' point in both the hands of both the genders of diabetic patients was fundamentally more extensive when contrasted with that of the controls. Contrasted with that of control, the mean tda edges in both the hands of both the genders of diabetics were restricted. They signify 'dat' edges were essentially thin just in the left hand of diabetic females when contrasted with left hands of female controls.

Burute et al. (2013) have meant to contemplate the different dermatoglyphic patterns in the patients of the development beginning diabetes mellitus (Type 2 diabetes mellitus) and contrasted and the dermatoglyphic patterns of controls. They did their investigation on 101 (51 male and 50 female) clinically analyzed patients of development beginning diabetes mellitus. For correlation, solid controls (Total $=100,50$ guys and 50 females) were considered. In female diabetics, essentially higher recurrence of arches and lower recurrence 
of whorls were seen than in controls. In diabetic female's outright finger ridge tally and complete finger ridge include were essentially lower than in controls. Discoveries of their examination feature on the potential markers to foresee type 2 diabetes mellitus on a bigger example size after a fastidious investigation of various fingertip dermatoglyphic factors.

Rakate and Zambare (2014) have looked at the distinctions on the fingertip patterns to be specific, curve, loop, and whorl in patients with type 2 diabetes mellitus with a control gathering. Test for their investigation included palmar prints of 350 type 2 diabetic patients old enough gathering between 30 and 60 years out of them 240 were guys and 110 were female contrasted and same age gathering of 350 non-diabetic people as control the gathering, out of the 240 were guys and 110 were females. In the example of 350 type 2 diabetes mellitus patients, they watched an expansion in the number of whorls in two hands of guys and females. The P-esteem was 0.001. The Ulnar loop and curve patterns were available in less incentive in diabetic patients which were measurably inconsequential. The plain whorl was available essentially higher in esteem in diabetic patients of guys and females. In diabetic guys in right hand 482 whorls where present. Yet, in charge just 326 whorls were available; this distinction was critical at 0.000 levels. In the left hand of diabetic, whorl was altogether more 418 than control 267, P-esteem was 0.000. Diabetic females additionally indicated higher recurrence of whorl pattern in two hands; on right hand 158 contrasted and control 121 and 184 on left hand contrasted and 130. The $p$ values were 0.010 on the right hand and 0.000 on the left hand. The Central pocket loop whorl pattern watched more in diabetic patients. In the diabetic male they 40 discovered 96 on the right hand and on the left hand 99 Central pocket loop whorl pattern which was more contrasted and control right hand 82 and left hand 82 . The P-values were 0.138 and 0.094 separately. In diabetic females additionally Central pocket loop whorl was watched all the righter hand 36, left hand 40 than control bunch right hand 29 and left hand 36; P-values were 0.237 and 0.320 . The twofold loop whorl was watched more in diabetic patients. In diabetic guys on the right hand, 54 twofold loop whorls were available which were more than control 38. Likewise, on left hand 69 in diabetic though 67 in charge. The P-values were 0.044 and 0.430 separately. In diabetic females, likewise, more recurrence of twofold loop whorl was seen on right hand 28 contrasted and control 15 and left hand 25 contrasted and 20 in charge. The P-values were 0.021 and 0.223 . At the point when they thought about a wide range of whorls together among diabetic and control gathering, noteworthy contrasts were seen in the two guys and females. In diabetic guys on the right hand, 632 whorls were available which were more than 446 in ordinary. Comparative discoveries were seen on left hand 586 in diabetic while 416 in charge. The P-values were 0.000 and 0.000 separately. In diabetic females, likewise, more recurrence of whorl was seen on right hand 222 contrasted and control 165. The $p$ values were 0.000 .

Karim et al. (2014) have looked at the distinctions in the fingerprint patterns and finger ridge include in patients with type 2 diabetic Mellitus with a control bunch in Erbil city, Kurdistan area, Iraq. In their examination, 50 non-insulin subordinate diabetes mellitus patients, 25 guys, and 25 females were contrasted and 50 (25 guys and 25 females) sound controls. The appropriation of fingertip patterns of male patients indicated no noteworthy contrast in ulnar loops, spiral loops and rose arches while plane arches expanded altogether $(p<0.05)$ in diabetic type 2 patients contrasted and controls, whorls diminished fundamentally $(p<0.05)$. Higher frequency of ulnar loops, spiral loops and plane arches in female diabetics contrasted and control females. They saw that essentially expanded $(p<0.05)$ center finger ridge include in the left hand of male diabetic patients. Fundamentally expanded $(p<0.05)$ file and little finger ridge check of the right hand was seen in female diabetic patients contrasted and control female groups. 
Bala et al. (2015) have considered an all-out 210 subjects out of which 70 subjects having diabetes (32 guys and 38 females), 70 subjects having diabetes with hypertension (32 guys and 38 females) and 70 typical sound people (32 guys and 38 females) as control having a place with Gangtok area of Sikkim. All were clinically analyzed and affirmed by examinations as diabetic and diabetic with hypertensive patients. In their investigation, an examination of diabetic with control bunch indicated the mean estimations of atd and dat edges in two hands of diabetic patients lower than control, though mean estimations of adt edges were higher than control bunch on both right and left sides. The huge distinction was found in the correct hands of diabetes mellitus gathering. In both right and left hands of males and females, the mean estimations of atd point and dat edge of the diabetic gathering were lower than in charge. The mean estimations of adt edge were higher than control. No noteworthy contrast was found. The mean estimations of a-b ridge include in two hands were higher in diabetic male and female aside from in the left hands of male and profoundly noteworthy distinction was found in both hands of the female.

Mehta and Mehta (2015) have analyzed fingertip patterns of type 2 diabetic patients with controls. One hundred type 2 diabetes mellitus patients (50 male and 50 female) were chosen for study and contrasted and equivalent number of controls. In two hands of guys and females' diabetic patients' frequency of whorls was essentially expanded. Frequency of loops was fundamentally diminished in two hands of male and female diabetics contrasted with controls. Arches were essentially diminished in both ways' hands of male diabetes mellitus patients. Arches were fundamentally decreased in left hand of female diabetics. In their investigation, they expressed that dermatoglyphics can be utilized as a screening device for the conclusion of people who are progressively inclined to create diabetes mellitus and, in this way, forestalling the future diabetic intricacies.

Bala et al. (2016) have examined a sum of 100 type 2 diabetic patients (50 guys and 50 females) were contrasted and 100 diabetics with hypertension patients of Hilly district. The mean estimations of all-out finger ridge check and total finger ridge include were higher in male and lower in female diabetic gathering than diabetic with hypertension gathering. The mean estimations of a-b ridge include were lower in males and higher in females in diabetic gathering and a critical contrast was found. The mean estimations of atd edge were higher in diabetic gathering than diabetic with hypertension gathering. The mean estimations of dat edge were lower in the right hands and higher in the left hands of diabetic gathering. The mean estimations of adt point were higher in guys and lower in female diabetic gathering than diabetic with hypertension gathering. In the right hands, the mean estimations of fingertip ridge include were lower in all digits aside from in 2nd, fourth, and fifth digits in the male diabetic gathering. In left hands, the mean estimations of fingertip ridge include were lower in all digits of diabetic gathering apart from in 2nd, fourth and fifth digits and no critical contrast were found. In their examination, they watched an expansion in ulnar loops in the correct hand of male diabetic and diminished frequency in the left hand of male and in two hands of female diabetics.

Tafazoli et al. (2013) collected samples for this exploration was gotten from 40 patients with acquired Essential hypertension disease and 20 ordinary subjects with no indications of Essential hypertension disease until 2 ages prior. Print of their palm and fingers was acquired in two groups by printing ink. Patterns of fingertips, ATD edges, A-B ridge and the various types of details were found, recognized and measured outwardly within four divisions of fingerprints. The consequence of this investigation indicated two patterns of fingertips; Whorl and curve in diseases are more than ordinary individuals. The most widely recognized tips in ordinary individuals is loop. In normal the quantity of the A-B ridge in female diseases and male-typical are individually more on normal than female 
ordinary and male sicknesses. The ATD point is lower in patients than in typical. Consequences of the overview on details demonstrated that frequency of the ridge Ending (E) is between with the most elevated frequency. The frequency of Bifurcation (B) is lower than the ridge finishing which it is between $\%$ in the left hands. Additionally, in some part of the fingers noteworthy distinction was found. 32-52.52 17.39-35 but in the right hand the second most patterns intermingling (C) which is between 14.13 and $38.66 \%$. The third most patterns in the left hand was seen in pattern unions $(\mathrm{C})$ with a rate between 8.62 and 32.6, however, in the right hand, the third most patterns are bifurcations (B), which is between 3.57 and $24.81 \%$.

The method proposed by Chakravathy et al. (2018) does observational examination included a correlation of palmar dermatoglyphic parameters among cases and controls. Parameters were dissected quantitatively-atd edge; subjectively outspread loop, ulnar loop, curve, whorl and composite. As appeared in (Graph I) out of all-out of 250 cases, 120 (48\%) were guys and 130 (52\%) were females. Of the absolute 250 controls, 137 (54.8\%) were guys and 113 (45.2) were females. As indicated by (Graph II), They found that signify "atd" edge was higher in cases than controls and there was the factually critical association of signifying "atd" point in cases contrasted and controls. They likewise discovered both ways signify "atd" point was higher in cases than controls with solid factually huge association of right signify "atd" edge in cases than controls. By dissecting subjective parameters in study gathering, conveyance of dermatoglyphic patterns was measurably noteworthy in situations when contrasted and controls. The outspread loop was increasingly visiting in cases while ulnar loop was more ordinarily found in normotensive controls. The examination of subjective parameters in each hand of cases and controls demonstrated an appropriation of dermatoglyphic parameters that were factually huge. As appeared, looking at subjective parameters in each finger of the two cases and controls for the conveyance of dermatoglyphic patterns indicated factually noteworthy association.

Lahiri et al. (2013) used digital and palmar dermatoglyphic investigation of 145 normotensive subjects and 131 hypertensive subjects was performed. The parameters utilized were advanced ridge pattern, complete ridge tally, and atd edge. The outcome demonstrated that the twofold loop whorl patterns are available with higher frequency in hypertensives. If there should be an occurrence of the hypertensive people, frequency of twofold loop whorl pattern and curve are $4.57 \%$ and $5.79 \%$ individually yet those are simply $0.44 \%$ and $1.33 \%$ in normotensives. In spite of the fact that the distinctions of occurrences of whorl just as ulnar loop between two groups are not all that obvious, yet factually huge $(p<0.05)$. The occurrences of explicit patterns in the hypertensive and the normotensive populace have appeared. The all-out ridge tally unmistakably expressed that it is horribly raised if there should be an occurrence of the hypertensive populace contrasted with the normotensive populace. The various estimations of Average Total Ridge Count of hypertensive and normotensive people have appeared with their comparing frequency. Subsequent to processing the remedied atd edge, a normal of the atd points of the two hands is made. The mean worth, most extreme and least qualities and model estimation of rectified atd edge (normal of both hands considered) in hypertensive gathering and normotensive gathering. The general pattern of this parameter is best comprehended by measurable examination ( $t$ trial) of all out arrangement of information which shows contrasts in estimations of atd edges of the hypertensive and normotensive gathering are of factual hugeness $(p<0.05)$.

Tafazoli et al. (2013) does the examination and conclude an observational, systematic and handy examination utilizing a case-control observational methodology with straightforward irregular testing and without substitution did on two groups of solid subjects and patients experiencing hypertension; individuals with no other explicit hereditary diseases 
which thus influence dermatoglyphic readings. that loops were the most widely recognized patterns on left digits in all patients (guys and females). From a factual perspective, there is an important distinction in frequency of various patterns on the left digit $4(p=0.05)$ between genders yet other left digits indicated no measurably noteworthy contrast. On the correct digits, loops were higher in rate in the two sexual orientations however no huge factual distinction was seen between sexes for frequency of various patterns on right digits $(p<0.05)$. The higher occurrence of whorls on digit II and of loops on different digits. Their is no huge distinction in the frequency of the patterns between two hands $(p<0.05)$. As illustrated, frequency in the dissemination of loops were 70 and $58.8 \%$ for the left hand and 63.3 and $66.7 \%$, for the correct submit guys and females, separately. The distinction between frequencies of patterns was not measurably huge on any of the two $h$ ands in both genders neither the right nor the left nor both frequency of loops on the left and the correct digits were $70-63.3 \%$ in guys and $57.8-66.7 \%$ in females, separately. The contrast between frequencies of the patterns was not factually critical on two hands of both genders. The information shows that the frequency appropriations of whorls in the control and test (hypertensive) bunch were 75 and $63 \%$ on the correct hand and 79 and $70 \%$ on the left hand of guys, individually. No factually critical contrast was seen between the frequency appropriation of patterns on two hands of guys in the two groups $(p=0.04, p=0.02)$ level of Whorl and Arch is more in charge bunch than intolerant gathering while the level of Loop is more in-understanding gathering than solid gathering in the two sexual orientations. The percent dispersion of whorls on digits of guys was as high as 77 and $66.5 \%$ in the control and the hypertensive gathering, individually. There has been no factually noteworthy contrast between two groups for frequency of patterns on digits in guys. level of whorl digit patterns in females was 53.7 and $62.5 \%$ in the benchmark group and in the hypertensive gathering, individually. There has been no factually huge distinction between the two female groups in the frequency of patterns on digits.

The dataset utilized and examination by Wanga et al. (2015) was gathered from the Behavior Risk Factor Surveillance System (BRFSS) of Centers for Disease Control and Prevention (CDC) and is openly accessible and downloadable from the BRFSS site. BRFSS is the world's biggest and persistently led phone-based wellbeing study in regard to social hazard factors, incessant wellbeing conditions and utilization of preventive administrations. Built-up in 1984 with 15 states taking an interest in the study, it has a long history in conduct and incessant disease observation. The essential point of BRFSS is to track and measure singular wellbeing conditions and hazard practices that add to the main source of high grimness and death rates in the grown-up populace who are matured 18 years and the older in United States. The review covers a wide scope of wellbeing hazard factors, preventive wellbeing practices and wellbeing conditions, including hypertension, diabetes and carcinoma related things. By gathering an assortment of data and sharing them with general society, BRFSS empowers scientists to examine the connections between interminable diseases and their hazard factors. We propose to anticipate hypertension just utilizing the surveys other than clinical test information, anthropometric information or hereditary information. Its adequacy exhibits the practicability of building up a hypertension observation framework for an enormous size of the populace in a non-obtrusive and prudent way. What's more, the outcomes from this examination might be utilized to manage the advancement of projects outfitted towards forestalling and relieving explicit hypertension chance elements. (2) They propose to coordinate calculated relapse examination and fake neural systems for synchronous hazard factor determination and hypertension prediction. In spite of the fact that theyt now, the proposed approach is basically a general system that can encourage specialists to break down other ceaseless diseases and different types 
of information. (3) They detail the choice of fake neural system engineering and the setting of applicable parameters, which is a troublesome and testing task in model learning. This can conceivably assuage analysts of the mind-boggling model determination issue and empower them to concentrate on the issues under scrutiny. (4) To manage the class irregularity issues, they propose a viable under-examining method. Based on a bunch calculation and choosing the delegate tests from each group in the extent of the group size, the proposed strategy can choose the most discriminative examples from the greater part class while making us lose the minimal measure of data.

Ravindranath et al. (2003) proposed qualitative dermatoglyphics involving identical fingerprint pattern, interdigital pattern, hypothenar pattern and palmar wrinkle was concentrated on 26 female and 11 male rheumatoid joint pain patients. Examination between understanding male and control male; and patient female and control female has been finished. 'Chi' square test was performed. In male patients, with hands together, arches were expanded, loops/whorls were diminished. Incomplete Simian wrinkle was fundamentally expanded. In the correct hand, patterns were expanded in the third interdigital zone. Then again, in female patients there was a noteworthy increment in whorls and diminishing in loops on the principal finger on both the hands, increment in arches on the third finger; the two arches and whorls on the fourth finger of left hand. The present examination has accentuated that dermatoglyphics could be applied as a symptomatic device to patients with rheumatoid joint pain. Mazumdar et al. found there is an association between rheumatoid joint pain and dermatoglyphics. The conceivable relationship amongst RA and dermatoglyphics may empower dermatoglyphics as a marker device in the analysis of rheumatoid joint pain. The present examination has been attempted to discover the likelihood that the fingerprints and palmprints assume a significant job in the analysis of rheumatoid joint pain. The frequency of twofold loop whorl in the rheumatoid joint pain bunch was seen in pointing finger and center finger of right hand and left hand separately (Mazumdar 2015). The ulnar loops were altogether present in right and little finger of the left hand of rheumatoid joint pain patients contrasted and control. The complete fingerprint ridges were progressively various in right and left hand of rheumatoid joint inflammation gathering.

Narayanan et al. (2017) investigation was a case-control concentrate with 60 cases with rheumatoid joint pain and 60 controls. The result appears out of the all-out 60 cases, $12(20 \%)$ were male and $48(80 \%)$ were females. Of the complete 60 controls, $12(20 \%)$ were guys and $48(80 \%)$ were females. The subjective parameters in female was a measurably critical increment in the number of whorls morally justified and left hands of female patients contrasted with the controls. There was a factually noteworthy reduction in the outspread loops in both the hands of female patients contrasted with the controls and the abatement is increasingly huge in the correct hand. There was no measurably huge distinction in the ulnar loop pattern circulation in either hand of female There was a factually critical increment in the finger ridge includes of right to deliver male patients contrasted with controls. There was a critical increment in the ridge check of patients. There was a factually noteworthy reduction in arches in the left hand of females with Rheumatoid Arthritis contrasted with the benchmark group. The left hand in male patients contrasted with the controls. Absolute finger ridge check (included ridge tally of both ways hand) was fundamentally expanded in male Rheumatoid joint pain patients contrasted with the controls. The ridge includes of the correct submit female patients was altogether higher than that of controls. The ridge includes left-hand fingers in female patients was fundamentally higher than that of controls. The complete finger ridge tally (right-hand ridge tally + left-hand ridge tally) was essentially expanded in female patients contrasted with controls. The expansion in all-out finger ridge include was increasingly huge in female patients contrasted with the 
male patients. There was no critical contrast in the pattern force in male patients contrasted with the controls. There was a measurably critical increment in the pattern power in the correct hand of females in examination with controls.

Rajangam et al. (2008) perform examination of male patients indicated a pattern towards criticalness for 'all-out finger ridge tally', centrality in left hand for 'total finger ridge check', and morally justified for ' $a-b$ ridge tally'. Then again, in the female patients, 'supreme finger ridge check' was seen as critical for the right hand and 'a-b ridge means' left hand. The watched contrasts between the male and female patients just as with that of the control might be a direct result of the expanded whorl pattern adding to two tallies and the width of the palm and fingers, along these lines a more noteworthy number of ridges might be available. Obviously, the detail of spreading the fingers and the palm, likewise should be remembered.

Hwang et al. (2004) concluded that the outspread loop and whorl were progressively visit while the curve and ulnar loop were less continuous; these attributes of the spiral loop and whorl were unmistakable in the correct hand and fifth finger. The frequency of the spiral loop was turned around in their left hands and the third fingers. The complete fingerprint ridges were increasingly various in the RA gathering. Contrasts of both palmprint ridges and palmprint point atd between the RA and the benchmark groups were not conspicuous, then again, palmprint ridges c-d was progressively various in the RA gathering. The shut wrinkle was progressively visit though open and meeting wrinkles were less incessant in the RA gathering. The typical wrinkle was less continuous though Simian and Sydney wrinkles were progressively visit in the RA gathering; the general attributes were unmistakable in their correct hands. The general attributes of Sydney's wrinkles were turned around in the left hands of female RA gatherings. The all-out level of palm wrinkle transversely was lower in the RA gathering; the attributes of the Sydney wrinkle were progressively conspicuous.

Swati and Sujata (2016) used conventional radiograph which has been a standard path for distinguishing the JSW in RA since bones are obviously noticeable in X-beam. Toward the beginning of the disease, $90 \%$ of the side effects of Arthritis are found in hands. Manual reviewing strategies are not ready to separate a little contrast in JSW figuring. Electronic appraisal of joint space has an effect in dynamic and observing the treatment of joint pain patients. The info hand X-beam picture of the typical hand is of $1000 \times 2000$ pixels, this picture is resized in the preprocessing step, resized picture goals is $500 \times 500$ pixels. The division utilizes ASM strategy, the quantity of emphases required is 1000 . The state of hand I followed flawlessly after the 1000 emphasis. The consequence of the ASM division after 900 emphasizes. To separate bone and non-bone districts binarization activity is performed. Figure 6 shows the aftereffect of Binarization. Binarization is finished by Otsu's calculation. At that point, the skeletonization of the paired picture is completed. Skeletonization brings about midline discovery it utilizes a diminishing procedure. At that point, the pinnacle and valley focuses are recognized along with the skeleton. The key focuses that is the specific joint area is followed by LLM. RA tolerant joint area estimation process is appeared. At that point the different factual highlights are determined like mean, middle, change. Joint area precision is determined by considering the number of exact joints recognized partitioned by the absolute number of joints that are 14 .

Gobikrishnan et al. (2016) collected patient's data with rheumatoid joint pain in knee locale with disease span short of what one year and ordinary people with no knee disease utilized for this investigation. What's more, this examination was endorsed by the institutional moral council. Absolutely 5 patients and 5 control subjects warm picture information was gathered. The mean period of patients utilized for the investigation was $40 \pm 10$. What's more, the 
disease length was $5 \pm 2$. The benchmark group mean age was $40 \pm 10$. The patients with no clinical proof of knee inclusion was dismissed for this examination. The information was gathered by directing a camp at SRM organization of clinical science. Before the picture obtaining method composed endorsement from the patient to take part in the investigation was taken. The patients who had knee torment other than rheumatoid joint inflammation were dismissed for this investigation. The highlights like Standard deviation, Mean, Skewness and kurtosis were extricated for patients and control subjects fragmented picture. The acquired outcome indicated essentialness. The standard deviation was beneath 10 for control bunch Above 10 for patients experiencing rheumatoid joint pain. Mean worth was underneath 5 for control gathering or more 5 for patients experiencing rheumatoid joint inflammation. Kurtosis was beneath 10 for control gathering or more 10 for patients experiencing rheumatoid joint inflammation and Skewness was underneath 10 for patients experiencing rheumatoid joint pain or more 10 for control gathering. The worth discovered higher for patients because of higher temperature variety.

\subsection{Practices used for blood group prediction}

The Table 1 shows the different methods of blood group identification, which includes some of the traditional as well as unusual those are build using electrical or electronics components such as diode, sensors. The few researchers tried a software-based approach by processing image of blood sample, but only few used method called fingerprint pattern analysis to predict blood group with limited accuracy because thy apply this method with traditional paper and ink as sample collection mechanism, so it not provide high accuracy. In current era of digitization there are several image (fingerprint) computation techniques which explore a greater number of features from fingerprint image which extends the accuracy of prediction process. As per literature, there are many methods are available for determination of blood group from those some having pros and cons, but the popular and most traditional one is to take blood sample of an individual and test it against various antibodies to determine blood type 3 to 5 min but it not convenient to the small children's and individual having blood phobia. The fingerprint having lots of potential which explore different unique patterns those may leads to identify blood group very quickly and accurately.

\subsection{Analysis and prediction of lifestyle-based diseases}

The Table 2 show various methods and dataset or samples which are used for analysis and prediction of lifestyle-based diseases such as Diabetes, Blood pressure/Hypertension, Rheumatoid arthritis. The diseases arise with age but not all humankind suffers from such agebased disease. The methods and dataset used to study age-based disease are limited only daily activity, X-ray samples and some are the techniques used after arrival of such diseases. The age, blood group, daily activity, lifestyle of individual and fingerprint patterns analysis helps researchers to generate indication or risk prediction in early age of an individual. 


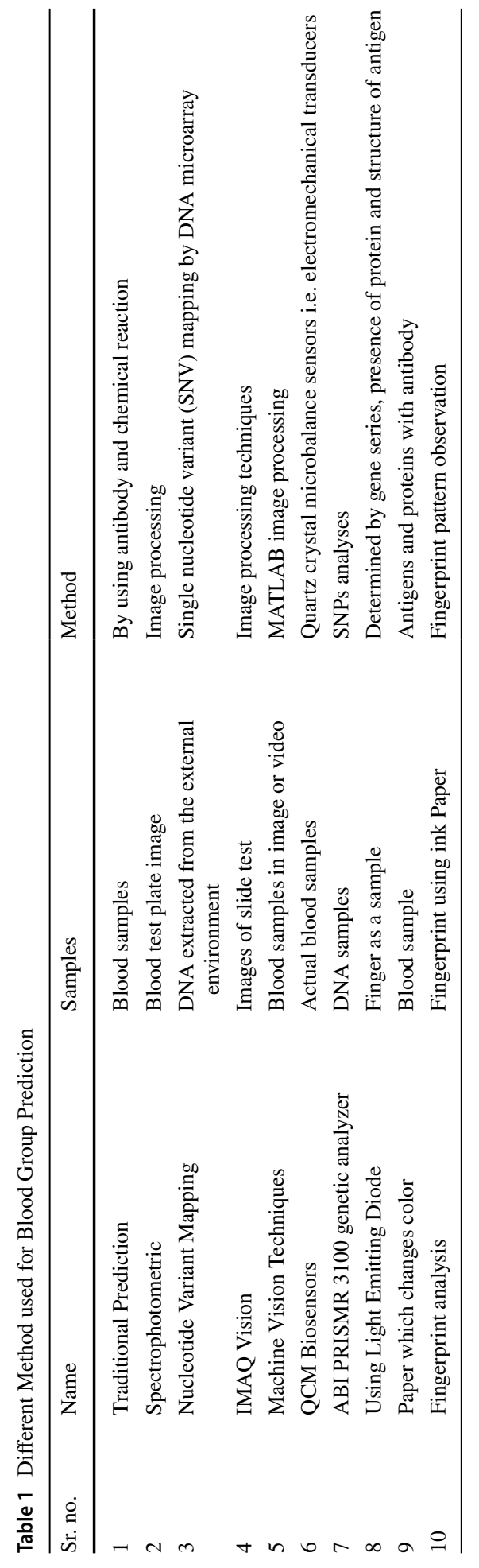




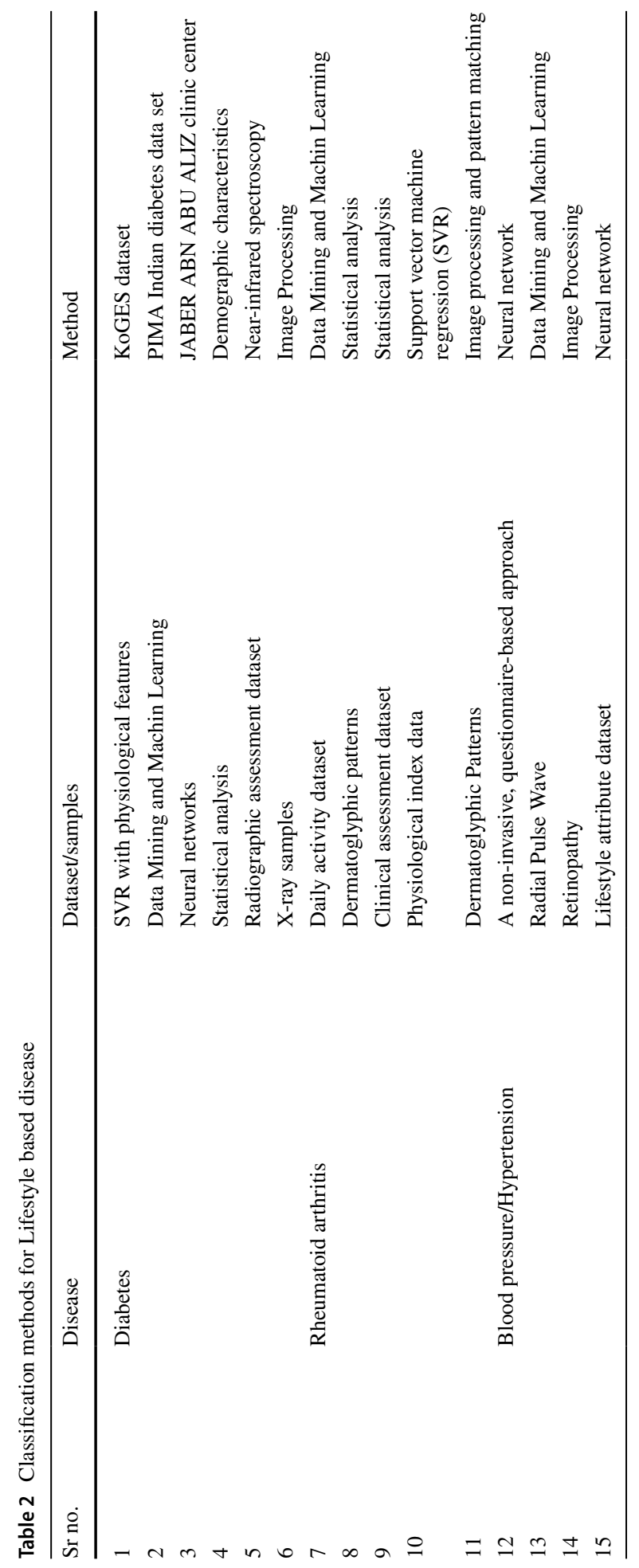




\section{Evaluation and discussion}

\subsection{Blood group}

As per literature, all authors attempt traditional method for sample collection as ink and paper, so they were only analyses the fingerprint patterns visible to human eyes those are like Loops, Arches and Whorls. Above literature shows relation between blood group and finger-print pattern summaries as follows:

- Loops were the determined common finger-print design and Arches were the least common.

- Whorls and mixed were moderate.

- More no of loops was originating in blood groups $\mathrm{O}, \mathrm{B}$ related to $\mathrm{A}$ and $\mathrm{AB}$.

- Blood group $\mathrm{O}^{+\mathrm{ve}}$ is the maximum found in samples, $\mathrm{O}^{-\mathrm{ve}}$ and $\mathrm{AB}^{-\mathrm{ve}}$ is the fewest.

- Loops, whorls, mixed and arches were uppermost in females.

- Group A was the utmost common group among sampled males.

- Blood group $\mathrm{O}, \mathrm{B}$, were the record usually seen in females.

\subsection{Diabetes}

In type $1 \mathrm{DM}$ there is increased frequency in whorls, and decreased ulnar loop, increased frequency of Sydney line, and increased incidences of arches in females (Ravendranath and Thomas 1995). In Maturity onset diabetes mellitus, there is decrease in mean value of TFRC, AFRC, increase in arches and decrease in whorls (Ravindranath et al. 2003). The fingerprint of an individuals with T2DM would be more irregular than an individual without T2DM, regulatory for gender and age. Diabetes regulate if wavelet analysis, a technique already used in forensics for fingerprint archival and matching, but not in previous studies of fingerprints as disease markers, would give results like the traditional ridge count or pattern analysis. The fingertips with whorls or double loops, applied a which RC formula that comprised half-unit values for those ridges situated between the core and delta point or between multiple cores. All ridge counting was as blinded to the diabetic and anthropometric status of the participants. The type 1 diabetes, there is increased frequency in whorls, and decreased ulnar loop, increased frequency of Sydney line, and increased incidences of arches in females (Roshani et al. 2016).

\subsection{Above literature shows relation between diabetes patients and finger-print pattern summaries as follows}

- Surge in arches in diabetes in both sexes

- Growth in rate of recurrence of loops and arches and a lessened frequency of whorls especially in mid finger

- Reduced number of arches in the right hand of male and left hand of female having diabetics, it was more in diabetic males and females than in the controls

- Growth in radial loop, ulnar loop in both male and female diabetics.

- Increase in frequency of whorls in both types of gender in diabetics 


\subsection{Blood pressure/hypertension}

There is increase in TFRC, decreased frequency of axial triradius ' $t$ ' in right palm of females and ' $t$ and $t$ ' in right palm of male, decreased atd angle and absence of axial of triradial in 10\% cases (Mandasescu et al. 1999). Above literature shows relation between patients having Hypertension and not a Hypertension finger-print pattern summary as follows:

- Higher prevalence of whorls and loops are associated with higher level of blood pressure

- Whorls and loops are prime ridge patterns in hypertensive patients

- ATD angle showed the mean of angle in patient surge rather than in control group

- Larger frequency of ridge endings in the thumbs and index fingers

- Amplified frequency in bifurcations and convergences in the middle, ring and little fingers

\subsection{Rheumatoid arthritis}

There is increase in arches and decrease in loops and whorls in males, whereas in females there is increase in whorls and decrease in loops on the 1st finger of both hands (Sengupta and Boruah 1996), with increase in arches on 3rd digit and whorls on 4th digit of left hand (Bala et al. 2015). Above literature study shows change in fingerprint patterns of patients having summarized as follows:

- Ulnar loop was the most prominent digital pattern in both genders,

- Decrease in the radial loop in both male and female patients

- Loops were significantly decreased in the third finger of males and a first and fourth finger of females

- Decrease in the ulnar loops in both the hands of male and female patients.

- Increase in the whorl pattern in the right hand of male patients and in both the hands of female patients

- Decrease in the arches of the left hand of female patients.

\subsection{Strengths and weaknesses of present research study}

The strength of the present research work is that fingerprint itself having lots of unique and hidden patents and it also currently used as a traditional, effective, and unique identification method of an individual. The dermatoglyphics as a diagnostic aid used from ancient eras and now it is well established in number of diseases which have strong hereditary basis and is employed as a method for screening for abnormal anomalies. There are more than 100 fingerprint minutiae patterns of ridges are determined as unique through the combination of genetic and environment factors.

The weaknesses are in acquisition of fingerprint and finding different unique patterns from people of different age group due to the human digital fingerprint varies in texture as person ages, so it is very difficult to classify fingerprints because there are the fingerprints having the characteristics of two or more patterns changes with age of an individual. Normally common clinical diseases like hypertension, arthritis and diabetes arises 
with aging, but due to busy schedule or lifestyle of an individual, it arises at any stage of life. So, it may lead to increase sample size and distribution of features required. The large datasets of fingerprint images acquired in real operational conditions are, rightly so, secured under data protection regulations that severely restrict the access to these data, even for research purposes.

\section{Conclusion}

The fingerprints are having immense potential to have an effective method of identification. In this research, it investigates the problem of blood group identification and analysis of disease those arises with aging or disease called as lifestyle-based like hypertension, type 2-diabetes and arthritis from fingerprint by analyzing their patterns correlation with blood group and age of an individual. With the literature review study, it is observed that fingers of an individual are having multiple unique patterns those are need to be extracted with computerized method with fingerprints image captured using digital device which allow to find known association of fingerprints patterns which may enhance the authenticity of the fingerprints in blood group identification and early indication of lifestyle-based diseases of an individual.

The fingerprint used as a traditional, effective, and unique identification method of an individual, in future it allows researchers to investigate with various diseases other than those are arised with age but also helps to explore different antibodies or reactive process of human body in several diseases. Also, similar study helps to predict the risk of any kind of diseases in early age of an individual. The analysis and classification of community based on age, blood group, fingerprint patterns and lifestyle diseases help to tackle any pandemic in future like COVID-19 in which mankind may suffer a lot having lifestylebased diseases like hypertension, type 2-diabetes.

\section{References}

Ali MMH, Mahale VH, Yannawar P, Gaikwad AT (2016) Fingerprint recognition for person identification and verification based on minutiae matching. In: 2016 IEEE 6th international conference on advanced computing, pp 332-339

Ameer Y, Zafar R, Abbasi AMH, Rasheed MA, Habib H, Salahuddin T, Warriach SA, Tariq A, Ahmed T (2014) Finger prints pattern variation in diabetic patients. PJMHS 8(1):162

Azhagiri R, Anitha M, Hemapriya J (2018) Analysis of left thumb print pattern among different human blood groups. Int J Anat Var 11:103-106

Bala A, Deswal A, Sarmah PC, Khandalwal B, Tamang BK (2015) Palmar dermatoglyphics patterns in diabetes mellitus and diabetic with hypertension patients in Gangtok region. Int J Adv Res 3(4):1117-1125

Bala A, Deswal A, Sarmah PC, Khandelwal B, Tamang BK (2016) Comparative study of dermatoglyphic patterns of diabetes mellitus and diabetic with hypertension patients of hilly region. Int J Sci Study 3(11):108-112

Berlitz PA, Claussen J, Doring JS, Drechsel H, North Off H, Gehring FK (2012) Rapid automated blood group analysis with QCM biosensors. In: 5th European conference of international federation for medical and biological engineering IFMBE proceedings, vol 37, pp 1039-1042

Burute P, Kazi SN, Vasanti V (2013) A role of dermatoglyphic fingertip patterns in the prediction of maturity onset diabetes mellitus (type II). IOSR J Dental Med Sci 8(1):01-05

Chakravathy PG, Shirali A, Chowta KN, Ramapuram JT, Madi D, Raj Singh Chouhan R (2018) A handy tool for hypertension prediction: dermatoglyphics. Indian Heart J 70:116. https://doi.org/10.1016/j. ihj.2018.07.007 
Dalvi A, Kumar Pulipaka H (2018) Determination of blood group using Image processing. Int J Sci Eng Res 9:24-27

Datasheet of DiamedDiaclon Anti-A (2008) Diaclon Anti-B. Diaclon Anti-AB, Cressiers/Morat

Deopa D, Prakash C, Tayal I (2014) A study of fingerprint in relation to gender and blood group among medical students in Uttarakhand Region. J Indian Acad Forensic Med 32:24-27

Desai SD, Hadimani GA (2013) Dermatoglyphics and health. Genet Anatom Karnataka 7(1):01-09

Fayrouz NE, Farida N, Irshad AH (2011) Relation between fingerprints and different blood groups. J Forens Legal Med 1:18-21

Fernandes JM, Soares FO, Minas G (2013) RH phenotypes analysis by spectrophotometry in human blood typing. In: Proceedings of the IEEE 3rd Portuguese meeting, pp 133-136

Fernandes J, Pimenta S, Soares FO, Minas G (2015) A complete blood typing device for automatic agglutination detection based on absorption spectrophotometry. IEEE Trans Instr Measur 64(1):112-119. https://doi.org/10.1109/TIM.2014.2331428

Ferraz A (2013) Automatic system for determining of blood type using image processing technique. IEEE 3rd Portuguese meeting in bioengineering (ENBENG), pp 20-23

Ferraz A(2013) Automatic system for determination of blood types using image processing techniques. In: 2013 IEEE 3rd Portuguese meeting in bioengineering (ENBENG)

Ferraz A, Carvalho V, Soares F (2010) Development of a human blood type detection automatic system. Proc Eng 5:496-499

Fingerprint Identification - Project 2, American University of Beirut Faculty of Engineering and Architecture Department of Electrical and Computer Engineering-Adaptive Filtering and Neural Networks

Gobikrishnan M, Rajalakshmi T, Snekhalatha U (2016) Diagnosis of rheumatoid arthritis in knee using fuzzy $\mathrm{C}$ means segmentation technique. International conference on communication and signal processing IEEE, April 6-8, 2016, IndiaSant SM, Vare AM, Fakhruddin S (1980) Dermatoglyphic traits in Diabetes mellitus. J Anat Soc India. 29: 43

Hwang S-B, Chung M-S, Park J-S, Suh C-H (2004) Dermatoglyphic characteristics of patients with rheumatoid arthritis. Korean J Phys Anthropol 18:313

Igbigbi PS, Msamati BC, Ng'ambi TM (2001) Plantar and digital dermatoglyphic patterns in Malawian patients with diabetes, hypertension and diabetes with hypertension. Int J Diabet Metabol 9:24-31

IMAQ (2004) IMAQ vision concepts manual. National Instruments, Austin

Jain V, Singh AK (2015) An approach for minutia extraction in latent fingerprint matching. Int J 6(1):51-58

Jha L, Das KD, Ahamad Z (2015) Fingerprint pattern examination of right hand thumb in relation to Blood Group. Janaki Med College J Med Sci 3:58-61

Joshi S, Garg D, Bajaj P, Jindal V (2016) Efficacy of fingerprint to determine gender and blood group. J Dent Oral Care Med 2(1):103. https://doi.org/10.15744/2454-3276.2.103

Kanchan T, Chattopadhyay S (2006) Distribution of fingerprint patterns among medical students. J Indian Acad Forens Med 28:65-68

Karim JK, Mohammed AL, Saleem A (2014) Dermatoglyphics study of fingerprints pattern's variations of a group of type II diabetic mellitus patients in erbil City. Zanco J Pure Appl Sci 26(4):11-16

Keerthana D, Ranganathan L (2017) Design and development of blood sample analyzer using intelligent machine vision techniques. Int J Eng Sci Comput 7:12017-12020

Lahiri A, Bandyopadhyay S, Adhya S, Ghosh S, Goswami S, Bhattacharya P (2013) A study on relationship between dermatoglyphics and hypertension. IOSR J Dental Med Sci 7(6):62-65

Mandasescu S, Richards B, Cadman J (1999) Detection of pre- diabetics by palmar prints: a computer study leading to a low-cost tool. In: XIV international congress of the federation for medical informatics. Dec 31, Germany, Manchester, GMDS: 2000

Mazumdar S (2015) A case study on dermatoglyphics in rheumatoid arthritis. Int J Sci Res 4:448-449

McBean RS, Hyland CA, Flower RL (2014) Approaches to determination of a full profile of blood group genotypes: single nucleotide variant mapping and massively parallel sequencing. Comput Struct Biotechnol J 11:147-151

Mehta AA, Mehta AA (2015a) Study of fingerprint patterns in type II diabetes mellitus. Int J Anat Res 2015:1046-1048

Mehta AA, Mehta AA (2015b) Study of fingerprint patterns in type II diabetes mellitus. Int J Anatomy Res 3(2):1046-1048

Mittal M, Lala BS (2013) Dermatoglyphics: an economical tool for prediction of diabetes mellitus. Int J Med Health Sci 2(3):292-297

Morris MR, Bjoern Ch, Ludwar ES, Mamo MN, Shubrook JH (2016) A new method to assess asymmetry in fingerprints could be used as an early indicator of type 2 diabetes mellitus. J Diab Sci Technol 10(4):864-871 
Narayana BL, Rangaiah YKC, Khalid MA (2016) Study of fingerprint patterns in relation to gender and blood group. J Evol Med Dent Sci 5(14):630-633. https://doi.org/10.14260/jemds/2016/144

Narayanan SK, Pais CC, Shenoy PK (2017) Use of palmar dermatoglyphics in rheumatoid arthritis: a case-control study. IAIM 4(12):70-76

Narkis Banu A, Kalpana V (2018) An automatic system to detect human blood group of many individuals in a parellel manner using image processing. Int J Pure Appl Math 118:3119-3127

Nezhad HR, Shah NM (2010) Application of dermatoglyphic traits for diagnosis of diabetic type 1 patients. Int J Environ Sci Dev 1(1):36-39

Pimenta S, Minas G, Soares FO (2012) Spectrophotometric approach for automatic human blood typing. In: Proceedings of the IEEE 2nd Portuguese meeting, pp 101-104

Pimenta S, Soares FO, Minas G (2012) Development of an automatic electronic system to human blood typing. In: Annual international conference of the IEEE engineering in medicine and biology society (EMBC), pp 2712-2715

Pramila Padmini M, Narasinga Rao B, Malleswari B (2011) The study of dermatoglyphics in diabetics of North Coastal Andhra Pradesh population. Indian J Fund Appl Life Sci 1(2):75-80

Radhika RH (2016) Pattern of fingerprints and their relation with blood groups. Indian J Basic Appl Med Res 5:744-749

Rajangam S, Ravindranth R, Shubha R, Nagesh HV, Johnson J (2008) Dermatoglyphics-quantitative analysis in rheumatoid arthritis. Anthropologist 10(3):233-235. https://doi.org/10.1080/09720 073.2008.11891054

Rakate N, Zambare B (2014) Fingertip patterns: a diagnostic tool to predict diabetes mellitus. Nat J Med Dental Res 2(3):49-53

Ramasubramanian MK, Alexander SP (2009) An integrated fiberoptic-microfluidic device for agglutination detection and blood typing. Biomed Microdev 11:217-229

Rastogi P, Pillai KR (2010) A study of fingerprints in relation to gender and blood group. J Indian Acad Forensic Med 32(1):11

Ravendranath R, Thomas IM (1995) Finger ridge count and fingerprint pattern in maturity onset diabetes mellitus. Ind J Med Sci 49:153-156

Ravindran G, Joby T, Pravin M, Pandiyan P (2017) Determination and classification of blood types using image processing techniques. Int J Comput Appl 157(1):12-16

Ravindranath R, Shubha R, Nagesh HV, Johnson J, Rajangam S (2003a) Dermatoglyphics in rheumatoid arthritis. Indian J Med Sci 57(10):437-441

Ravindranath R, Shubha R, Nagesh HV (2003b) Dermatoglyphics in rheumatoid arthritis. Ind J Med Sci $57: 437-441$

Relf CG (2003) Image acquisition and processing with LabVIEW. CRC, Boca Raton

Roshani S, Pallavi I, Prabhakar S, Amita S (2016a) Comparative study on the dermatoglyphic pattern among diabetic (type-2) and non-diabetic adults in North Indian population. Eur J Pharm Med Res 2016:430-433

Roshani S, Amita S, Prabhakar S, Bezbaruah NK, Anshu M (2016b) Dermatoglyphic patterns among type 2 diabetic adults in North Indian population. Int J Curr Med Pharma 2(8):609-611

Sangam MR, Ramesh Babu A, Krupadanam K, Anasuya K (2011) Finger print pattern in different blood groups. J Indian Acad Forensic Med 67:343-345

Satoh K, Itoh Y (2004) Forensic ABO blood grouping by 4 SNPs analyses using an ABI PRISMR 3100 genetic analyser. Int Congr Ser 1288:49-51

Satoh K et al (2001) Evaluation of ABO subtyping by DNA sequencing. Jpn J Leg Med 55:111

Sengupta S, Boruah J (1996) Finger dermatoglyphic patterns in diabetes mellitus. J Hum Ecol 7(3):203206. https://doi.org/10.1080/09709274.1996.11907220

Sharma MK, Sharma H (2012) Dermatoglyphics: a diagnostic tool to predict diabetes. J Clin Diagn Res 6(3):327-332

Shivaleela C, Hanji CV, Kumar GV (2013) Utility of dermatoglyphics in Type II diabetes mellitus (T2DM) to assess the risk for IHD: Apilot study. Biomed Res 24(2):242-244

Shivhare PR, Sharma SK, Ray SK, Minj A, Saha K (2017) A study of dermatoglyphic pattern in relation to $\mathrm{ABO}, \mathrm{Rh}$ blood group and gender among the population of Chhattisgarh. Int J Sci Study 4:61-65

Singh M, Majumdar O (2015) Dermatoglyphics: blueprints of human cognition on fingerprints. Comput Sci Electron J 6:124-146

Siva Sundhara Raja D, Abinaya J (2019) A cost-effective method for blood group detection using fingerprints. Int J Adv Study Res Work 2:2581

Smail HO, Kareem SS, Abdulkareem NM (2019) Comparative study of the fingerprint pattern among diabetic (type 1) \& non-diabetic children in Koya City. J Adv Laborat Res Biol 10(2):41-47 
Subrahmanyam BV (1999) Modi"s medical jurisprudence and toxicology, 22nd edn. Butterworths, New Delhi, pp 71-77

Sudikshya KC, Maharjan N, Adhikari N, Shrestha P (2018) Qualitative analysis of primary fingerprint pattern indifferent blood group and gender in Nepalese. Hindawi Anat Res Int 2018:7

Sumathi S, Desai SD (2007) Study of dermatoglyphics in patients with type II diabetes mellitus essential hypertension in the age group between 35-55 Years. Anal Med 10(2):22-28

Swati AB, Dr. Sujata NK (2016) Automatic Analysis of Rheumatoid arthritis based on statistical features. In: 2016 International conference on automatic control and dynamic optimization techniques (ICACDOT). International Institute of Information Technology (I $\left.{ }^{2} I T\right)$, Pune, 2016 IEEE

Tafa Z, Pervetica N (2015) An intelligent system for diabetes prediction. In: 4th Mediterranean conference on embedded computing Budva, Montenegro pp 378-382

Tafazoli M, Dezfooli SR, Shahri NM, Shahri HM (2013a) The study of dermatoglyphic patterns and distribution of the minutiae in inherited essential hypertension disease. Curr Res J Biol Sci 5(6):252-261

Tafazoli M, Dezfooli SR, Kazemi T, Shahri HM, Shahri NM (2013b) Comparison of dermatoglyphic patterns between healthy and hypertensive people. Asian J Med Sci 5(1):19-25

Taiwo IA, Adebanjo OO (2012) Evaluation of association between digital dermatoglyphic traits and type-2 diabetes in Lagos, Nigeria. Niger Quart J Hospital Med 22(3):191-199

Tarca A, Tuluc E (2005) Dermatoglyphics in insulin: dependent diabetes or diabetes mellitus type 1 (T1 DM). J Prevent Med 13(1-2):43-53

Tejaswini HV, Mallikarjuna Swamy MS (2014) Determination and classification of blood types using image processing techniques. ITSI Trans Electr Electron Eng 2:2320-8945

Thakar MK, Sharma T (2016) Digital grid method for fingerprint identification and objective report writing. Egypt J Forens Sci 6:194-201

Udoaka AL, Lawyer-Egbe K (2009) Dermatoglyphic patterns of diabetic mellitus patients of Ijaw Origin in Port Harcourt, Nigeria. Niger J Health Biomed Sci 8(2):72-82

Umana UE, Bello R, Timbuak J, Ibegbu A, Musa SA, Ikyembe D, Hamman WO (2013) Dermatoglyphic and cheiloscopic patterns among diabetic patients: a study in Ahmadu Bello University Teaching Hospital Zaria, Nigeria. J Biol Life Sci 4(2):206-214

Veena Vijayan V, Anjali C (2015) Prediction and diagnosis of diabetes mellitus -a machine learning approach. In: 2015 IEEE recent advances in intelligent computational systems (RAICS), pp 10-12

Vij K (2005) Textbook of forensic medicine and toxicology, 3rd edn. Elsevier, New Delhi, pp 89-91

Wanga A, An N, Chen G, Li L, Alterovitz G (2015) Predicting hypertension without measurement: a noninvasive, questionnaire-based approach. Expert Syst Appl 42:7601-7609

Publisher's Note Springer Nature remains neutral with regard to jurisdictional claims in published maps and institutional affiliations. 